\title{
Inhibition of histone acetyltransferase function radiosensitizes CREBBP/EP300 mutants via repression of homologous recombination, potentially targeting a gain of function
}

\author{
Manish Kumar 1,13, David Molkentine (1) 2,13, Jessica Molkentine (1) 2,13, Kathleen Bridges ${ }^{3}$, Tongxin Xie ${ }^{4}$, \\ Liangpeng Yang (1D ${ }^{3}$, Andrew Hefner ${ }^{2}$, Meng Gao ${ }^{4}$, Reshub Bahri (iD ${ }^{2}$, Annika Dhawan (D) 2, Mitchell J. Frederick ${ }^{5}$, \\ Sahil Seth (10) 6, Mohamed Abdelhakiem (10) ${ }^{2}$, Beth M. Beadle7, Faye Johnson (1) 8,9, Jing Wang (1) 9,10, Li Shen ${ }^{10}$, \\ Timothy Heffernan (1) ${ }^{6}$, Aakash Sheth ${ }^{11}$, Robert L. Ferris ${ }^{12}$, Jeffrey N. Myers ${ }^{4,9}$, Curtis R. Pickering ${ }^{4,9,13}$ \& \\ Heath D. Skinner (1) 2,13凶
}

Despite radiation forming the curative backbone of over $50 \%$ of malignancies, there are no genomically-driven radiosensitizers for clinical use. Herein we perform in vivo shRNA screening to identify targets generally associated with radiation response as well as those exhibiting a genomic dependency. This identifies the histone acetyltransferases CREBBP/EP300 as a target for radiosensitization in combination with radiation in cognate mutant tumors. Further in vitro and in vivo studies confirm this phenomenon to be due to repression of homologous recombination following DNA damage and reproducible using chemical inhibition of histone acetyltransferase (HAT), but not bromodomain function. Selected mutations in CREBBP lead to a hyperacetylated state that increases CBP and BRCA1 acetylation, representing a gain of function targeted by HAT inhibition. Additionally, mutations in CREBBP/EP300 are associated with recurrence following radiation in squamous cell carcinoma cohorts. These findings provide both a mechanism of resistance and the potential for genomically-driven treatment.

\footnotetext{
${ }^{1}$ Department of Biochemistry, All India Institute of Medical Sciences (AIIMS), Bilaspur, Himachal Pradesh, India. ${ }^{2}$ Department of Radiation Oncology, University of Pittsburgh, UPMC Hillman Cancer Center, Pittsburgh, PA, USA. ${ }^{3}$ Department of Experimental Radiation Oncology, University of Texas, MD Anderson Cancer Center, Houston, TX, USA. ${ }^{4}$ Department of Head and Neck Surgery, University of Texas, MD Anderson Cancer Center, Houston, TX, USA. ${ }^{5}$ Department of Otolaryngology-Head \& Neck Surgery, Baylor College of Medicine, Houston, TX, USA. ${ }^{6}$ TRACTION Platform, University of Texas, MD Anderson Cancer Center, Houston, TX, USA. ${ }^{7}$ Department of Radiation Oncology, Stanford University, Stanford, CA, USA. ${ }^{8}$ Department of Thoracic and Head and Neck Medical Oncology, University of Texas, MD Anderson Cancer Center, Houston, TX, USA. ${ }^{9}$ The University of Texas Graduate School of Biomedical Sciences, Houston, TX, USA. ${ }^{10}$ Department of Biostatistics, University of Texas, MD Anderson Cancer Center, Houston, TX, USA. ${ }^{11}$ Department of Medicine, Baylor College of Medicine, Houston, TX, USA. ${ }^{12}$ Department of Otolaryngology, University of Pittsburgh, UPMC Hillman Cancer Center, Pittsburgh, PA, USA. ${ }^{13}$ These authors contributed equally: Manish Kumar, David Molkentine, Jessica Molkentine, Curtis R. Pickering, Heath D. Skinner.

凶email: skinnerh@upmc.edu
} 
W ith a few isolated exceptions, the cure of solid tumors requires effective local therapy. In the vast majority of disease sites, this translates to a need for radiation, either alone or as part of a treatment package, including surgery. Despite the large number of targeted and immunotherapies introduced over the past decade or more, in nearly all cases, the only agents available to improve responses to radiation are the cytotoxic chemotherapies that have been in use since the 1980s or earlier. Because of this, an effective and minimally toxic radiosensitizer has the potential to, in short order, positively impact hundreds of thousands of patients.

One exemplar of this phenomenon is head and neck squamous-cell carcinoma (HNSCC), the curative treatment of which has remained largely unchanged over the past two decades. While $~ 75 \%$ of patients with HNSCC require radiation for the treatment of their disease, the recent failure of cetuximab means there are generally no biologically driven radiosensitizers available to improve response and decrease toxicity of this therapy ${ }^{1-4}$. Nor has the advent of immunotherapy changed the current paradigm, with a recent clinical trial of chemoradiation combined with immunotherapy closed due to lack of efficacy ${ }^{5}$.

Despite these failures, the search for improved combinations with radiation remains critical. Again, focusing solely on the close to 300,000 patients with HNSCC annually worldwide who are recommended to receive radiation, an agent that improves the efficacy of this treatment by $15 \%$ could lead to more than as 30,000 lives saved annually ${ }^{6,7}$.

However, most antineoplastic agents tested in the preclinical setting ultimately fail to be translated to the clinic due to multiple factors, including the artificial nature of in vitro systems and unforeseen toxicity ${ }^{8,9}$. Targets identified as radiosensitizers in an in vitro model, may underperform in vivo due to complex interactions within the tumor itself. Additionally, the same microenvironment interactions could be potential targets for radiosensitization and may not be readily identified using in vitro screening techniques.

Additionally, most large-scale screening approaches using cell lines with known genomic status have not exposed the cells to radiation and, thus, have not identified tumor mutations or alterations that may be associated with specific targets for radiosensitization ${ }^{10}$. The model of a genomically mediated "Achilles' heel" in tumors harboring a specific genotype has perhaps best been characterized for BRCA1-altered tumors and their dramatic response to PARP inhibition ${ }^{11}$. However, this model can be expanded to the combination of novel agents with DNA-damaging therapies in specific genetic backgrounds ${ }^{12}$. This is highly advantageous as genomically driven radiosensitizers have the potential to only affect the mutated cancer cells, and not normal cells, providing improved responses with less toxicity.

In the current investigation, we begin with an in vivo screening analysis to identify radiosensitizers in HNSCC, as well as a further analysis to identify potential targets based on somatic mutation. These data lead to the identification of histone acetylation as a target, potentially driven by a gain of function in certain classes of HAT/TAZ2-domain mutants. These mutations exhibit reduced basal-inhibitory function, leading to a hyperacetylated state and potential dependency on homologous recombination and BRCA1 for DNA-damage repair. The importance of CREBBP and EP300 mutation is underscored following analysis of tumor tissues in several cohorts of patients with SCC of the head and neck, lung, or cervix treated with radiation therapy, identifying these mutations as associated with radioresistance and poor outcome.

\section{Results}

In vivo screening identifies multiple potential radiosensitization targets. We performed in vivo shRNA library screens in tumors generated from 5 HNSCC cell lines of varying genetic background and HPV status (HPV-positive: UM-SCC-47 and UPCI:SCC-152), (HPV-negative: UM-SCC-22a, HN31, and Cal 27) treated with radiation (Supplementary Table 1). Two libraries were used: one encompassing most genes known to be targeted by available antineoplastic agents currently in clinical use or in clinical trials and the second targeting the DNA-damage repair pathway (Supplementary Table 2).

To determine a gene-level summary estimate of the impact of knockout of each gene, we performed redundant shRNA analysis (RSA) to generate $\log p$-values for each gene for irradiated tumors (Fig. 1a). Additionally, quantile-transformed median fold change (fc) for each target was analyzed (Fig. 1b). This analysis identified several targets known to be associated with radiosensitization, such as CDK6, XIAP, PIK3CA, and PTK2 $2^{13-17}$. However, to further identify targets specific for radiation response, as opposed to those primarily inhibiting tumorigenesis, we evaluated RSA $-\log \mathrm{p}$ values for irradiated compared with untreated tumors in complimentary experiments reported elsewhere ${ }^{18}$ (Fig. 1c, d). We examined the average for each group and selected targets highlighted in orange in Fig. 1d, based on values favoring radiation response versus effects on tumorigenesis, defined very broadly to maximize potential target identification (Supplementary Table 3 for selected targets). In this group, we identified multiple DNA-damage repair genes, such as TP53BP1, ATRIP, and RUVBL1. Additionally, inhibition of XIAP and PIK3CA was highly associated with radioresponse, even above their antitumor effects.

Gene ontology (GO) analysis of targets identified in Fig. 1d was then performed (Fig. 1e) and, as expected based on the targets screened, DNA-damage repair processes were highly represented in this analysis. However, several additional pathways, particularly protein lysine acetylation, were also identified.

CREBBP, EP300, or dual-specificity protein-kinase (TTK) inhibition in combination with radiation in CREBBP/EP300mutated tumors leads to radiosensitization. In addition to finding general radiosensitization targets, we wished to determine if specific somatic mutations observed in HNSCC were associated with targets, with a goal of identifying genomically associated radiosensitizers. To accomplish this, we compared the existing RSA log $p$-values and median fold change data for radiosensitizing targets between tumors that are wild type or mutant for somatic mutations that are represented by the models in the study. This was defined as at least 2 of 5 models harboring a mutation that is observed in $>10 \%$ of HNSCC. The specific comparisons were: (i) CREBBP/EP300 (HN31 and CAL 27 vs. UM-SCC-47, UPCI:SCC-152 and UM-SCC-22a), (ii) NOTCH1 (HN31, UM-SCC-47, and UM-SCC-22a vs. CAL 27 and UPCI:SCC-152), and (iii) CASP8 (UM-SCC-47, UM-SCC-22a and CAL 27 vs. HN31 and UPCI:SCC-152) (Fig. 2a-c).

While no differences were observed in the comparison of CASP8 wild-type and mutant tumors in either of the libraries, several targets seemed to preferentially increase sensitivity to radiation in NOTCH1 and CREBBP mutant tumors (Fig. 2a and Supplementary Fig. 1). Inhibition of Abl1, CCNO, and KDM1a appeared to preferentially radiosensitize NOTCH1 mutant tumors (Supplementary Fig. 1), while inhibition of the CREBBP and EP300 genes, as well as the dual-specificity protein kinase (TTK), was associated with increased in vivo sensitivity to radiation in the CREBBP mutant tumors in this screen (Fig. $2 \mathrm{a}-\mathrm{c}$ ). 
a.

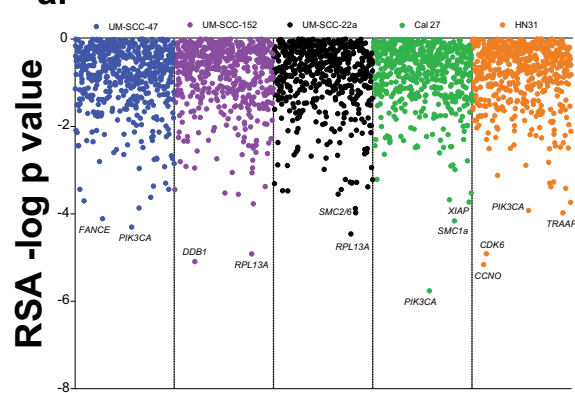

d.



b.

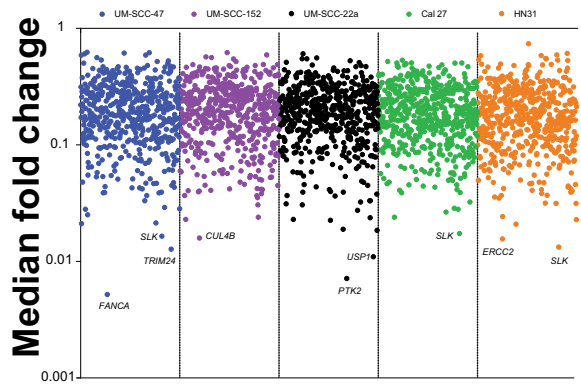

e.
C.

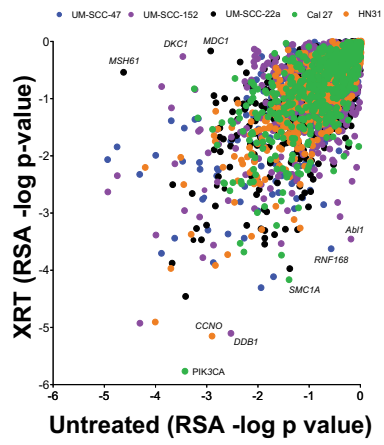

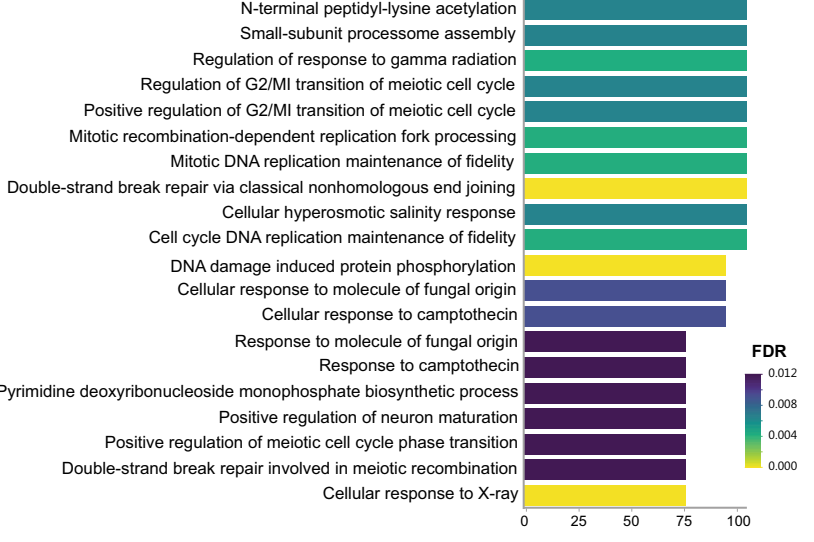

Fig. 1 In vivo shRNA screening for radiosensitizing targets. a \& b RSA -log $p$-values (a) and median fold change (b) for each target in our two shRNA screening libraries for each tumor type tested. $\mathbf{c} \& \mathbf{d}$ RSA - $\log p$-values in irradiated (XRT, y axis) versus untreated ( $x$ axis) for each tumor type tested (c) and the average for each group (d). Target genes in orange were selected for further analysis as potential radiosensitizers and analyzed for Gene Ontology term enrichment (e) (two-sided $p$-value presented).

Inhibition of CREBBP or EP300 expression leads to in vitro radiosensitization, but only in the presence of cognate mutations. Based on the degree of effect as well as the identification of lysine acetylation as an enriched pathway in radiosensitizing targets, we chose CREBBP and EP300 for further validation by performing targeted knockdown (KD) across an expanded set of cell lines. We utilized shRNA KD to either CREBBP or EP300 in HNSCC cell lines (Fig. 2d and Supplementary Fig. 2) of varying CREBBP/EP300 mutation status (see Supplementary Table 1 for details). These cell lines were then treated with radiation, and clonogenic survival was assayed (Fig. 2d). Similar to our in vivo screening results, CREBBP or EP300 KD was associated with increased sensitivity to radiation. However, the sensitivity appeared only in the context of a mutation in the cognate gene. For example, $C R E B B P \mathrm{KD}$, but not EP300, led to significant radiosensitization in the CREBBP mutant cell line UM-SCC-22a, which is EP300 wild type. Similarly, EP300 KD, but not CREBBP, led to significant radiosensitization in the EP300 mutant cell line UM-SCC-25 that is wild type for CREBBP. This pattern was consistently observed over all cell lines tested (Fig. 2d).

CREBBP inhibition leads to increased apoptosis and decreased BRCA1 foci formation following radiation in mutant cells. To further evaluate the observed radiosensitization, we examined apoptosis in multiple cell lines expressing shRNA to CREBBP (Fig. 3a, b). The combination of CREBBP inhibition and radiation led to dramatically increased TUNEL staining in CREBBP mutant (but not wild type) cell lines (Fig. 3a). Similar results were observed on immunoblot examining caspase- 3 cleavage (Fig. $3 b$ ). We also examined the DNA-damage response via immunofluorescence staining of DNA-damage foci (Fig. 3c) (representative images in Supplementary Fig. 3a). Importantly, $\mathrm{y}$ $\mathrm{H} 2 \mathrm{AX}$ foci, a marker of DNA damage, were increased following $C R E B B P \mathrm{KD}$ and radiation in all 3 CREBBP mutant cell lines examined. In contrast, under the same conditions, BRCA1 foci induction was significantly reduced in all 3 CREBBP mutant cell lines and $\mathrm{HN} 30$ (Fig. 3c). In the HN30 line $\mathrm{\gamma}-\mathrm{H} 2 \mathrm{AX}$ induction was reduced following radiation and $C R E B B P \mathrm{KD}$, likely indicating a different biology occurring in this TP53 and CREBBP WT cell line. Irrespective of mutational status, inhibition of CREBBP generally had little effect on 53BP1 foci formation following radiation (Fig. 3c). CREBBP inhibition also had minimal effects on BRCA1 transcription or cell cycle distribution at baseline or following radiation, regardless of mutation status (Supplementary Fig. 3b-d).

Knockdown of CREBBP leads to dramatic in vivo radiosensitization. We further evaluated the therapeutic potential of targeting CREBBP using three separate in vivo models of HNSCC, two harboring mutant $C R E B B P$ and one with wild-type $C R E B B P$. In the first study, we used the CREBBP mutant cell line UM-SCC47 to generate tumors in the mouse flank. Tumors were treated with 2 Gy $x 8$ days, in a fractionation scheme designed to recapitulate that used in patients, albeit to a much lower dose (16 Gy total vs. $70 \mathrm{~Gy}$ in the clinic). In this experiment, radiation or CREBBP KD (using two distinct shRNA constructs) alone had minimal-to-modest effect; however, the combination led to a profound tumor-growth delay, decreased tumor volume, and improved survival (Fig. $4 \mathrm{a}, \mathrm{b}$ ). Indeed, at the conclusion of the tumor growth delay experiment, seven tumors in the irradiated shCREBBP-2 group (64\%) and three tumors in the irradiated shCREBBP-3 group (21.4\%) had regressed below the limits of 

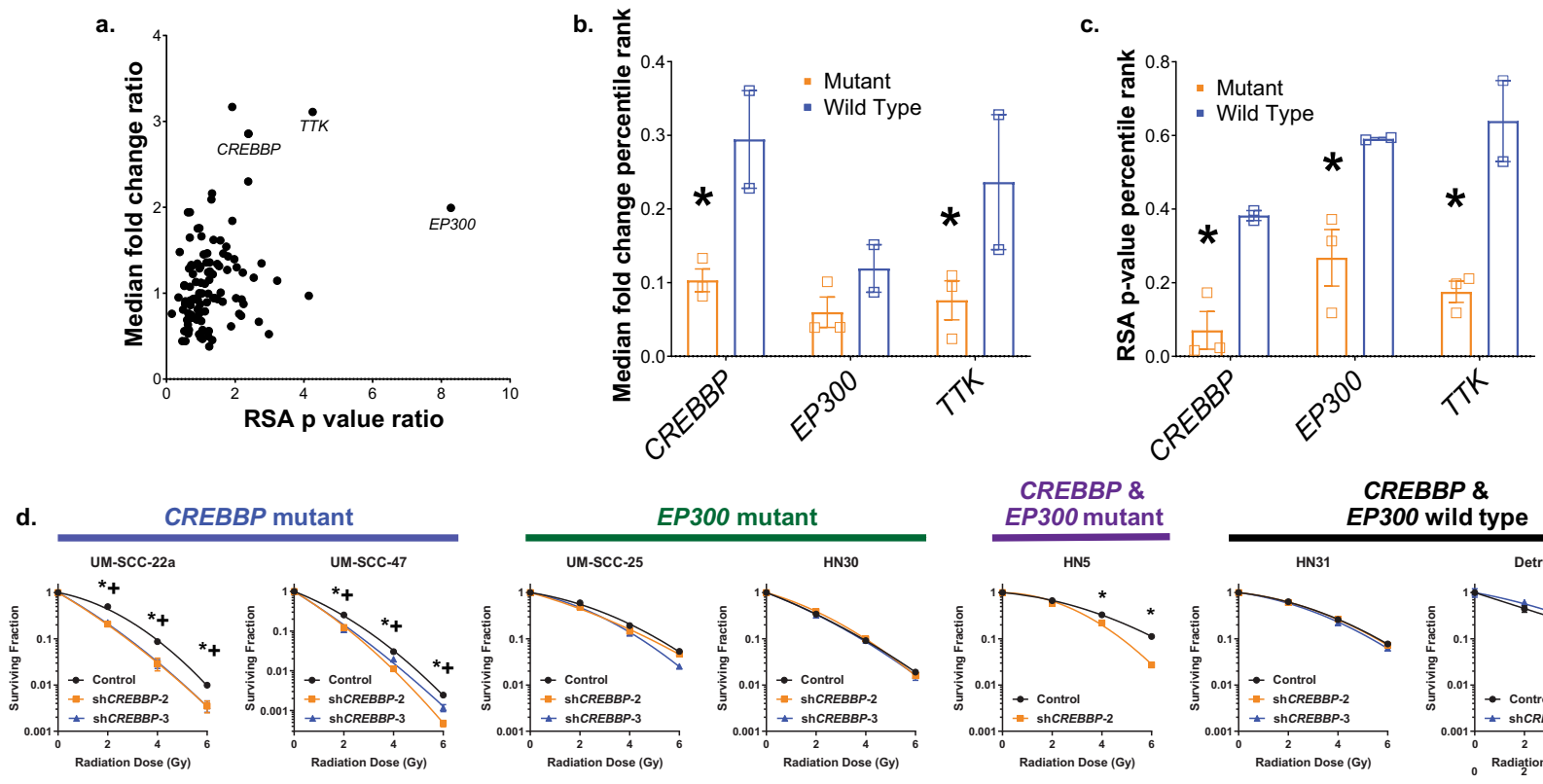

CREBBP \&
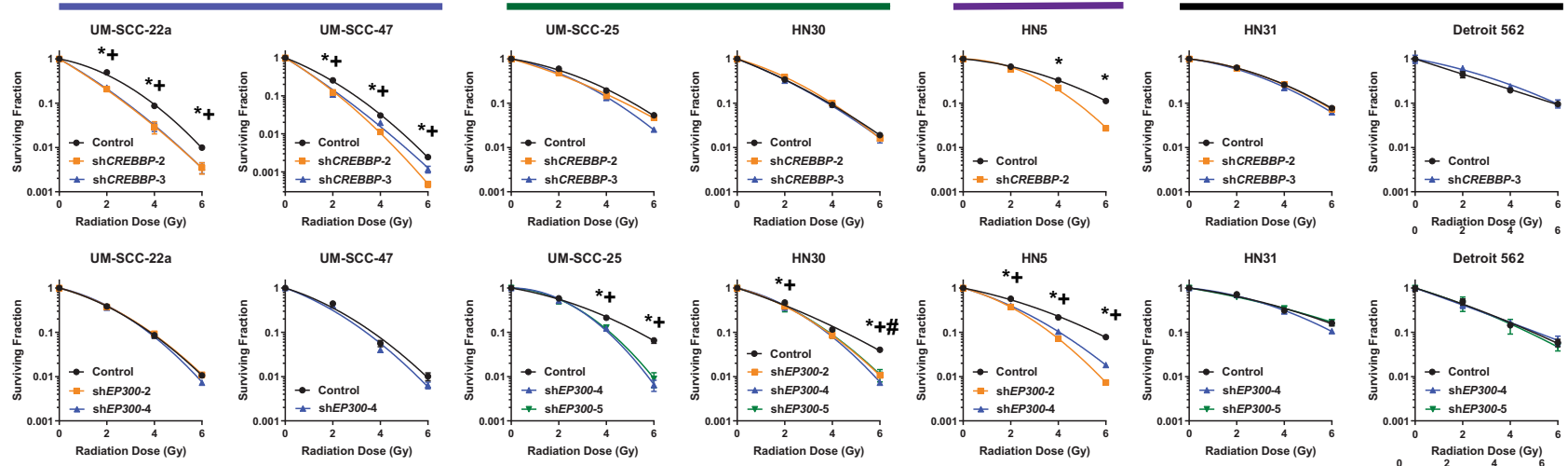

Fig. $\mathbf{2}$ In vivo screening identifies genomically associated radiosensitization in CREBBP/EP300-mutated tumors. a Ratio of CREBBP mutant vs. wild type for target fold change ( $y$ axis) and RSA log $p$-value ( $x$ axis) for radiosensitizing targets selected from Fig. 1. b, c Difference between CREBBP/EP300 mutant $(n=3)$ and wild-type $(n=2)$ tumors from the in vivo shRNA study as a function of target fold change (c) and RSA log $p$-value $(\mathbf{c})$. $\left(^{\star}\right)-$ two-sided $p<0.05$ versus wild type. $\mathbf{d}$ Clonogenic assays following irradiation of HNSCC cell lines expressing control and either CREBBP or EP300 shRNA using a minimum of 3 independent samples for each condition and are presented as mean values $+/-$ SEM. In B-D, comparisons were evaluated using ANOVA with post hoc analysis adjusted for multiple comparisons. For $\left(^{\star}\right)$ shCREBBP-2 and (\#) shCREBBP-3, $p<0.05$ versus control. All $p$-values two-sided.

detection. An additional separate experiment was performed to measure apoptosis. TUNEL staining increased in the combined shCREBBP and radiation groups $8 \mathrm{~h}$ following the final dose of radiation ( $2 \mathrm{~Gy} \times \mathrm{x}$ d) compared with both the irradiated shControl tumors and unirradiated CREBBP knockdown tumors (Fig. 4b). We performed a similar experiment using tumors derived from cells of another CREBBP mutant line, UM-SCC-22a (Fig. 4c). While inhibition of CREBBP alone had a significant effect in this model, we again observed a profound radiosensitization compared with the unirradiated control tumors. In this model, virtually all tumors dramatically regressed, and most tumors regressed below the limits of detection.

We additionally performed a similar experiment using tumors derived from the CREBBP wild-type cell line Detroit 562 (Fig. 4d). In this model, treatment with $2 \mathrm{~Gy}$ x 8 days led to significant tumor-growth delay in the control and shCREBBP tumors. However, inhibition of $C R E B B P$ led to no significant radiosensitization. Specifically, tumor-growth delay was slightly (although not significantly) worse with CREBBP knockdown, with no effect on final tumor volume.

Inhibition of CBP and p300 histone acetyltransferase (HAT), but not bromodomain function, leads to radiosensitization in $C R E B B P / E P 300$ mutants associated with repression of HR. To further examine the therapeutic relevance of the observed radiosensitization in HNSCC, we utilized several chemical inhibitors of CBP and/or p300 function: (1) ICG-001, a CBP-specific inhibitor that is thought to inhibit the interaction between CBP and $\beta$-catenin, although it is also known to have $\beta$-catenin- independent effects (note: PRI-724 is an active enantiomer of ICG-001) ${ }^{19-21}$; (2) GNE-272, a bromodomain-specific inhibitor for both CBP and p30022; (3) A-485, a histone acetyltransferase inhibitor specific for CBP and p300 (note: A-486 is an inactive analog and used as a negative control $)^{23}$. Similar to shRNA-based inhibition of $C R E B B P$, ICG-001 led to significant in vitro radiosensitization on clonogenic assay, but only in those cell lines harboring a CREBBP mutation (Supplementary Fig. 4a, b). Although the analog of ICG-001, PRI-724, is actively in clinical trial development, neither of these agents target p300, and as predicted, we generally did not observe similar sensitization in a wild type or an EP300 mutant cell line (Supplementary Fig. 4a, b).

Thus, to maximize the clinical impact of the observed radiosensitization, we examined additional inhibitors, currently in clinical development, that inhibit both CBP and p300 function. We initially tested GNE-272, a bromodomain inhibitor, however, this agent had minimal effects on sensitivity to radiation on clonogenic assay (Supplementary Fig. 5), independent of CREBBP or EP300 status. However, the HAT inhibitor A-485, but not the inactive A-486 analog, led to a profound radiosensitization in cell lines harboring a mutation in either CREBBP or EP300 (Fig. 5a, c), but not in wild-type cell lines (Fig. 5b, c). The observed radiosensitization was largely due to increased apoptosis following the combination of A-485 and radiation (Fig. 5d, e).

Because of the observed effects of shCREBBP on BRCA1 foci formation following radiation, and the relationship between BRCA1 and homologous recombination (HR), we wished to evaluate the relationship between HAT inhibition and homologous recombination directly via I-Scel-based assay ${ }^{24}$. We 

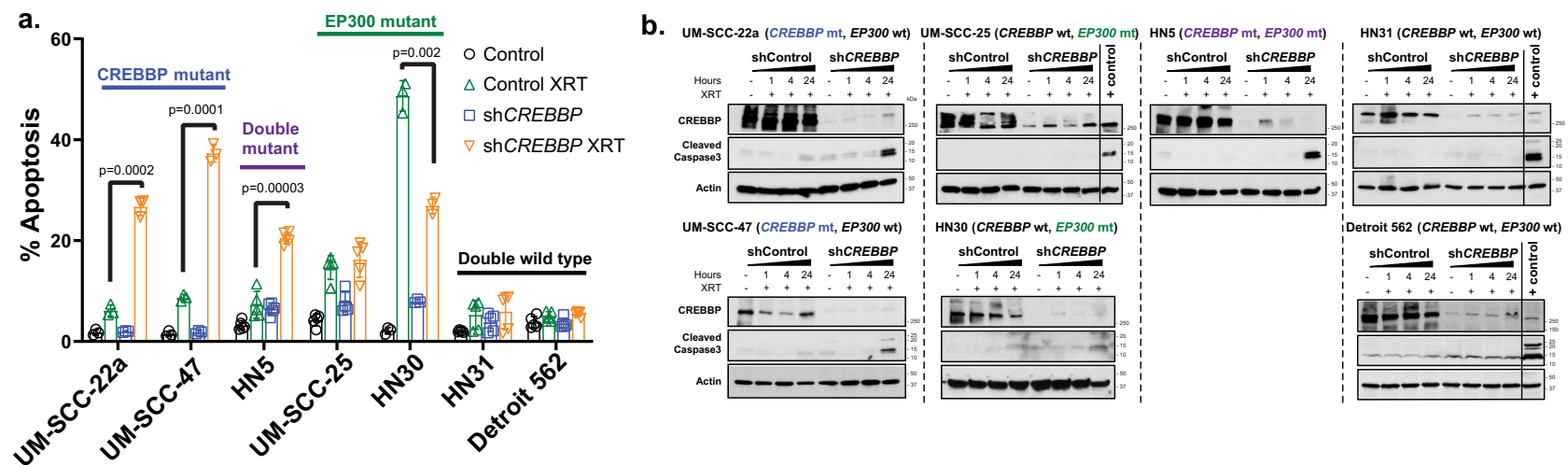

c.
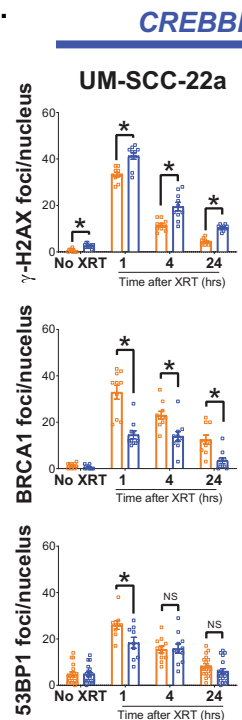

REBBP mutant

UM-SCC-47
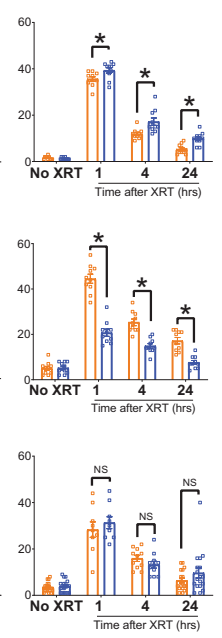
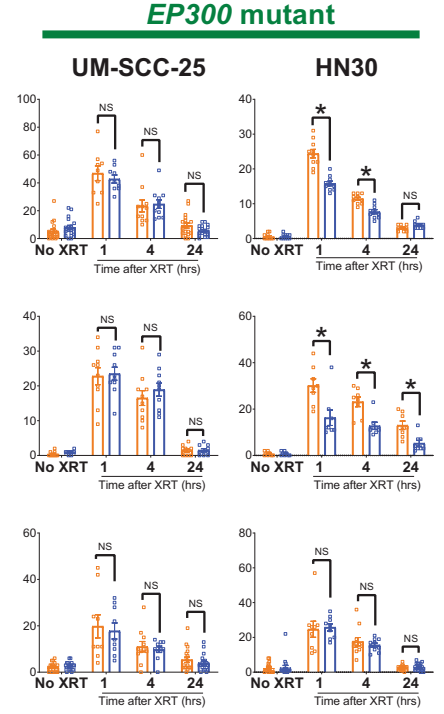
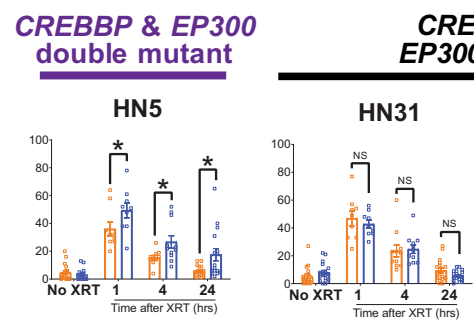

CREBBP \&

EP300 wild type
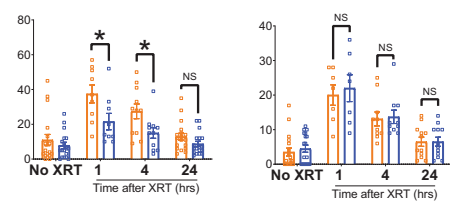

Detroit 562
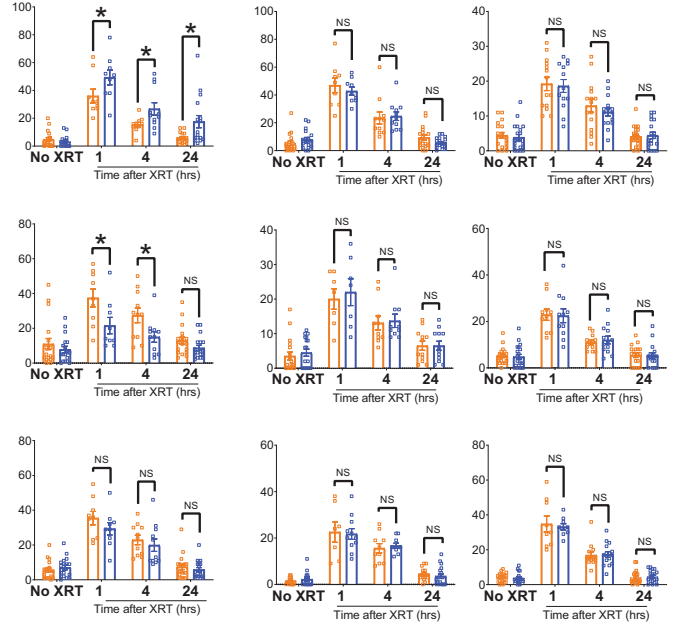

Fig. 3 Impaired HR and increased apoptosis is observed following CBP inhibition in CREBBP mutant cells. a, b TUNEL staining (a) and caspase-3 immunoblot (b) following irradiation in HNSCC cells expressing control or CREBBP shRNA. c $\mathrm{\gamma}-\mathrm{H} 2 \mathrm{AX}, 53 \mathrm{BP} 1$, and BRCA1 foci following irradiation in shControl or shCREBBP HNSCC cells. Significance tested via ANOVA with post hoc analysis adjusted for multiple comparisons. For $\mathbf{a}$, c, a minimum of three independent samples for each condition are shown and are presented as mean values +/- SEM. For A, two-sided p-value shown for each indicated comparison. For $\mathbf{c}_{,}\left(^{*}\right)$ indicates a two-sided $p<0.05$ for the comparisons shown.

utilized both HNSCC and lung-cancer cell lines (which also harbor mutations in either CREBBP or EP300) (Supplementary Table 1). Similar to HNSCC lines, treatment with A-485 generally led to more profound radiosensitization in CREBBP/EP300 mutant but not wild-type lung-cancer cell lines (Supplementary Fig. 6a, b). Additionally, A-485 led to dramatic and significant repression (ranging from $44 \%$ to $75 \%$ versus control) of $\mathrm{HR}$ in CREBBP or EP300 mutant HNSCC and lung-cancer cell lines, but not in wild-type cells (Fig. 6a) (Supplementary Fig. 6c, d). Conversely, A-485 had little effect on NHEJ in HNSCC cell lines (Fig. 6b).

The observed radiosensitization in CREBBP/EP300 mutants is not CREBBP or EP300 expression-level dependent. One potential explanation for the observed radiosensitization is a simple dose-dependency. Namely, if basal levels of CREBBP or EP300 are significantly diminished in mutant cells (and tumors), a more profound inhibition is possible, leading to a more pronounced phenotype. Thus, the observed effect could be due to a more complete inhibition of the protein in the setting of a loss-offunction mutation. To further explore this hypothesis, we examined basal expression in HNSCC cell lines and tumors in the context of various $C R E B B P$ and $E P 300$ mutations. Interestingly, basal CREBBP and EP300 gene expression in the cell lines used in this study was not directly associated with the underlying mutation (Supplementary Fig. 7a). Moreover, in a panel of 82 HNSCC cell lines, neither CREBBP nor EP300 mutation was directly associated with mRNA expression ${ }^{25}$ (Supplementary Fig. 7b).

In clinical samples from the TCGA HNSCC cohort, no significant difference in CREBBP (Supplementary Fig. 7c) or EP300 (Supplementary Fig. 7d) gene expression was observed in mutant tumors. Moreover, even in the context of nearly complete inhibition of CBP protein expression (Fig. 3b), neither HN31 nor HN30 (CREBBP wild-type cell lines) were sensitized to radiation (Fig. 2d). Conversely, incomplete inhibition of EP300 (in the case of EP300 mutants HN5 and HN30) led to significant radiosensitization (Fig. 2d and Supplementary Fig. 2). Collectively based on these data, the presence of radiosensitization in mutant tumors (and its absence in wild-type tumors) does not appear to be directly related to total amount of either CREBBP or EP300 present.

Histone acetylation is associated with radiosensitization in CREBBP/EP300 mutants following $\mathrm{CBP} / \mathrm{p} 300$ targeting in HNSCC. Based on the observed sensitization following HAT, but 
a.

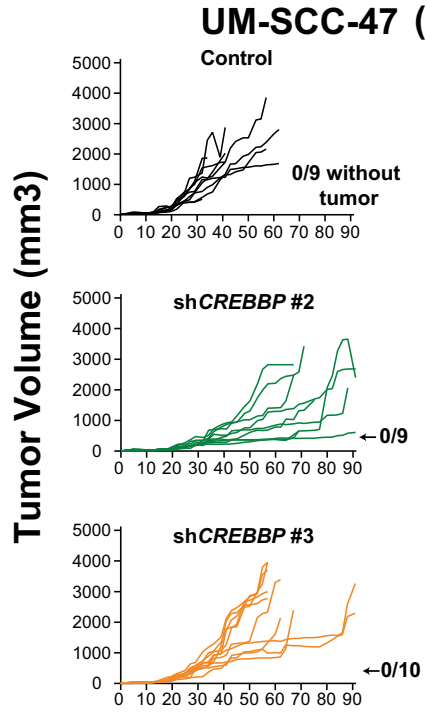

\section{Days post-injection}

C.

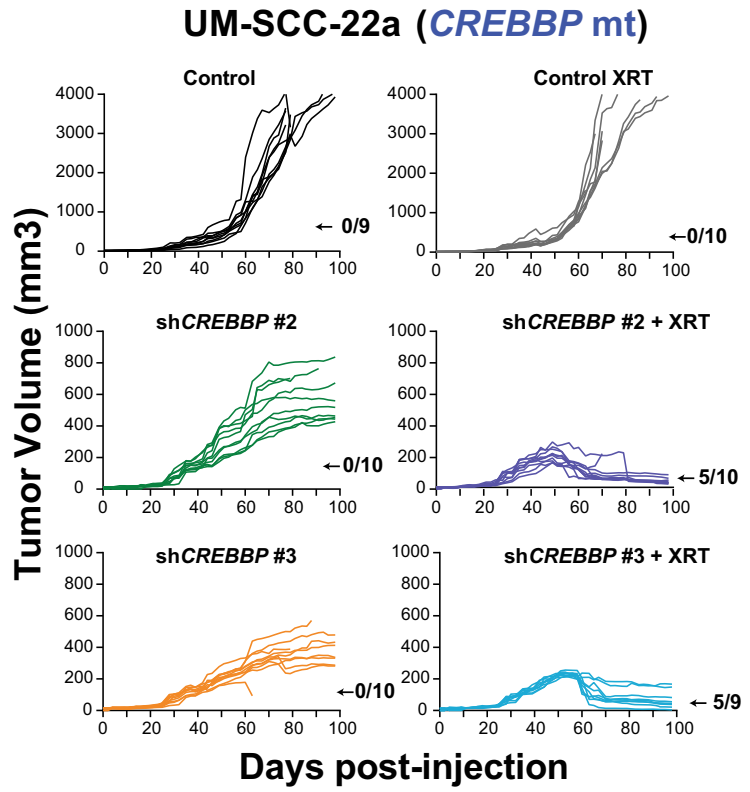

Tumor growth delay
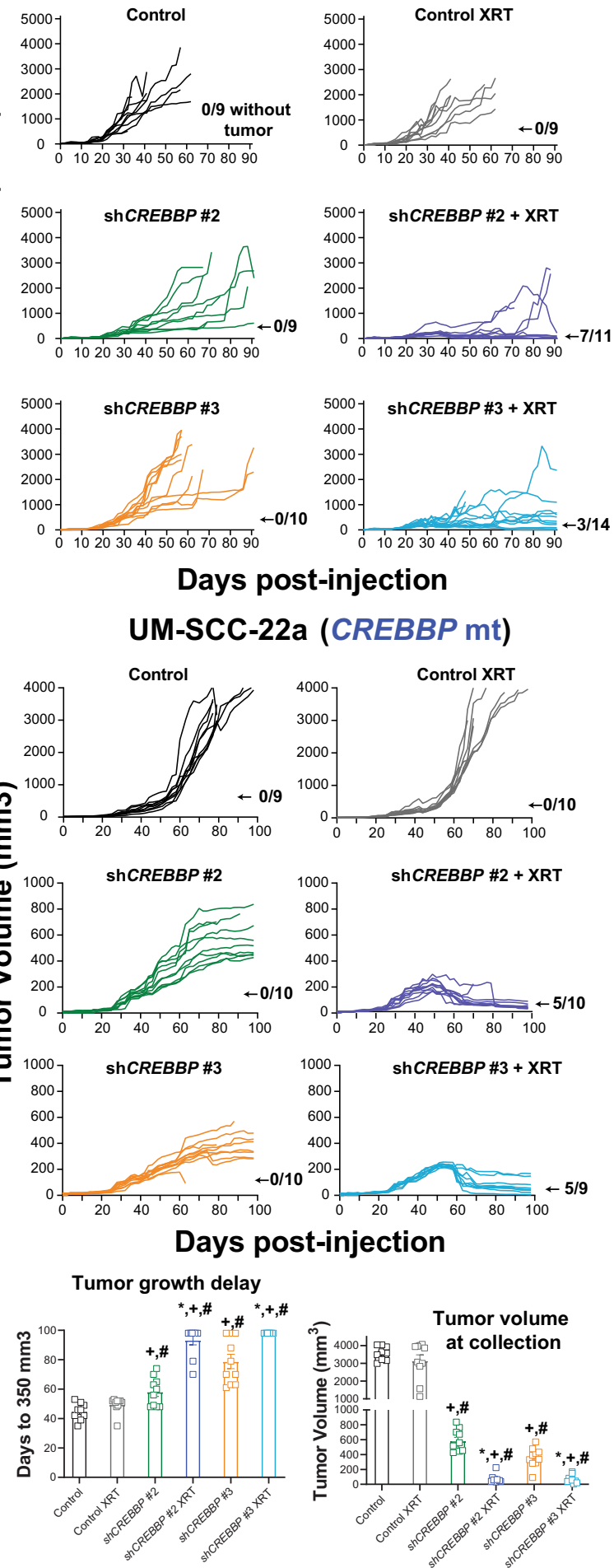

$0 \underset{0102030405060708090}{10}$

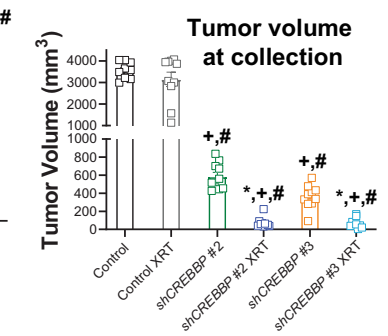

b.
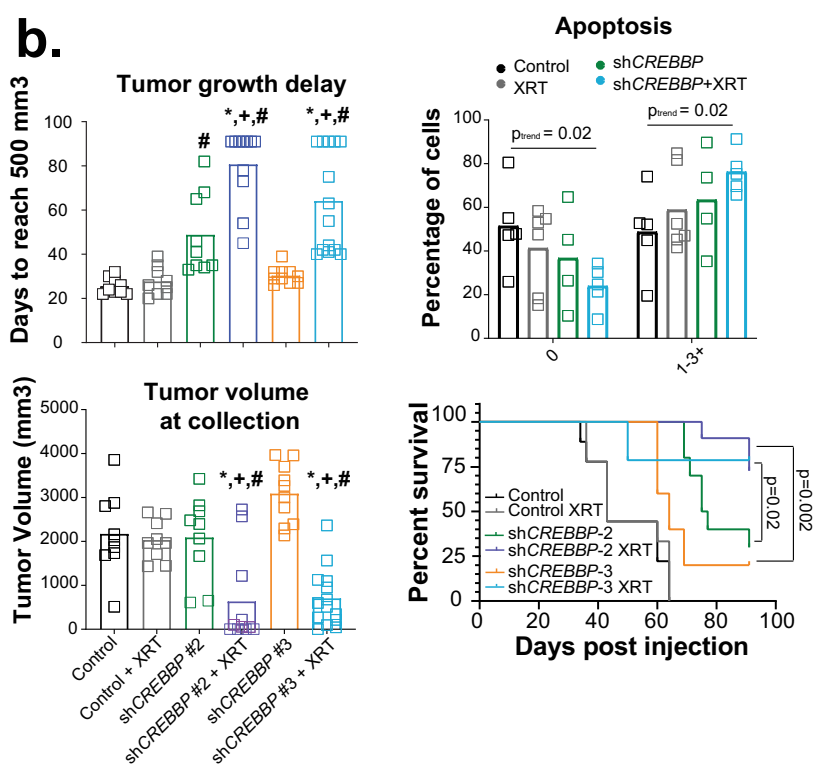

\section{d. Detroit 562 (CREBBPIEP300 wild type)}
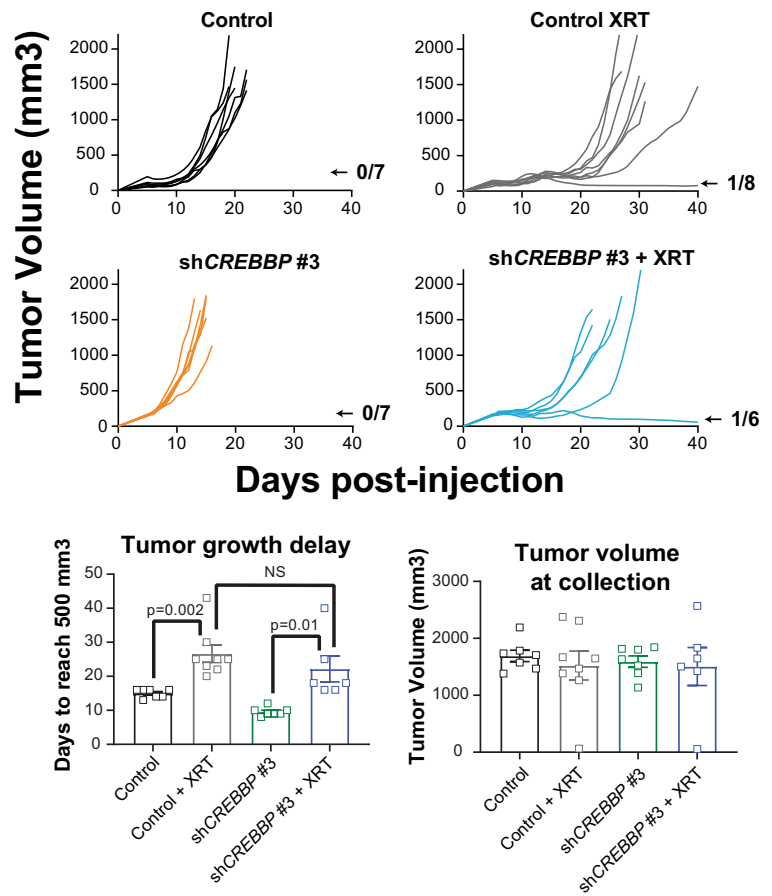

Fig. 4 Inhibition of CBP in CREBBP mutant tumors leads to radiosensitization in mutant but not wild-type in vivo HNSCC models. a Individual tumorgrowth curves in a UM-SCC-47 xenograft model expressing either control or two different CREBBP shRNAs following irradiation at 2 Gy/day for eight days. b Tumor-growth delay (to $500 \mathrm{~mm} 3$ ), tumor volume at collection, survival (all with $\mathrm{n}$ per group shown in panel a), and in vivo apoptosis (via TUNEL assay) ( $n=$ minimum of 4 ) in UM-SCC-47 xenograft tumors. Tumors for TUNEL assay were collected $8 \mathrm{~h}$ following the final dose of radiation in a concurrent experiment with tumor growth delay and survival. Log-rank statistics comparing shCREBBP-2 XRT or shCREBBP-3 XRT groups versus unirradiated control for each group were performed (two-sided $p$-value shown). c Individual growth curves, tumor-growth delay, and tumor volume at collection from a similar experiment performed in the UM-SCC-22a xenograft model. Of note, tumor-growth delay ( $n$ as shown in the tumor growth panel) to 350 mm ${ }^{3}$ was used for this experiment as many tumors in the treatment groups did not reach $500 \mathrm{~mm}^{3}$. d Individual growth curves, tumor-growth delay, and tumor volume at collection ( $\mathrm{n}$ for both shown in the growth-curve panel) from a similar experiment in the Detroit 562 xenograft model. For bar graphs, data are presented as mean values +/- SEM. Significance tested via ANOVA with post hoc analysis adjusted for multiple comparisons. Two-sided $p<0.05$ as indicated or compared with unirradiated $\operatorname{shCREBBP}\left({ }^{\star}\right)$, unirradiated control $(+)$, irradiated control (\#). 
a.

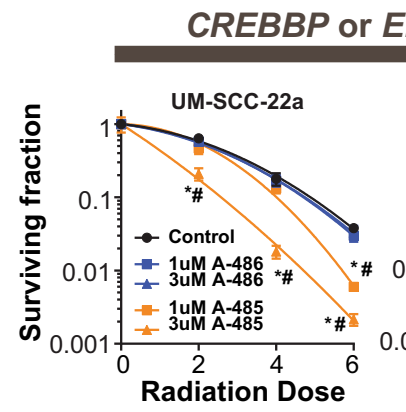

HN30


e.

\section{b. CREBBP \& EP300 wild type}


c.

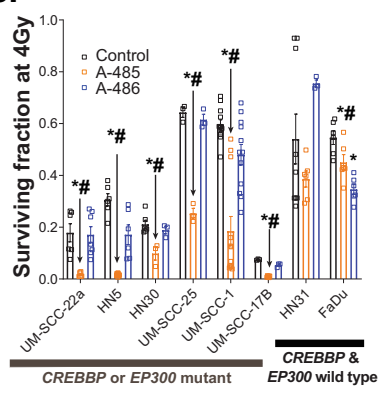

CREBBP or EP300 mutant

CREBBP \& EP300 wild type

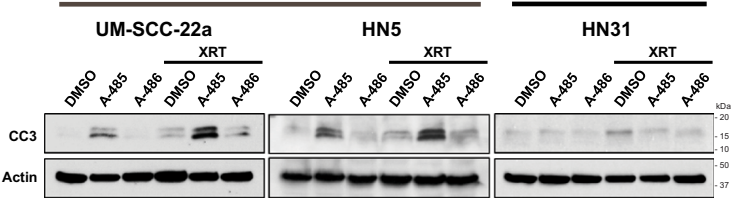

Fig. 5 The HAT inhibitor A-485 radiosensitizes cells harboring CREBBP and EP300 mutations. a, b Clonogenic survival following irradiation and either A-485 (active) or A-486 (inactive) in CREBBP/EP300 mutant (a) or wild-type (b) HNSCC cells. c Surviving fraction at 4 Gy for each cell line (highest dose of A-485/A-486 shown). d, e TUNEL assay (d) and caspase 3 cleavage (e) in UM-SCC-47 (CREBBP mut), and HN5 (CREBBP/EP300 mut) and HN31 (CREBBP/EP300 wt) cells treated with radiation and either A-485 (active) or A-486 (inactive). For a-e, a minimum of three independent samples for each condition are shown and are presented as mean values $+/-$ SEM with $\left({ }^{\star}, \#\right)-p<0.05$ vs. control $\left({ }^{*}\right)$ and A-486 (\#). p-values are two-sided and derived from ANOVA with post hoc analysis adjusted for multiple comparisons.

not bromodomain inhibition, and the lack of evidence for this phenomenon being solely gene-expression dependent, we further investigated histone-acetylation status in HNSCC cell lines following combination treatment. As expected, treatment with A-485 inhibited histone acetylation at H3K18 and H3K27 in HN5 and UM-SCC-22a cells (Fig. 7a). Both cell lines harbor mutations in either CREBBP and/or EP300 and exhibit sensitivity when HAT inhibition is combined with radiation. Interestingly, $\mathrm{FaDu}$, a CREBBP/EP300 wild-type cell line with no observed radiosensitization, showed no effect on histone acetylation following treatment with HAT inhibitor at baseline or following radiation treatment. Similar effects on histone acetylation were observed following the expression of shRNA specific to CREBBP (Fig. 7b) and treatment with ICG-001, although a slight decrease in acetylation was observed in $\mathrm{FaDu}$ cells following this latter treatment (Fig. 7c).
Selected mutations in CREBBP exhibit increased acetylation activity consistent with gain of function and potentially mediating response to radiation. Previously, mutations within the inhibitory TAZ domain of CBP have been linked to increased histone acetylation ${ }^{26}$. Because of this observation, we evaluated two of our cell lines (UM-SCC-22a and UM-SCC-17b) with similar mutations (Fig. 8a, b). Compared with wild-type cells, these cell lines exhibited profoundly higher levels of acetylation of several histone marks, as well as increased global protein acetylation (Fig. 8a). Additionally, CBP protein itself was acetylated at higher levels compared with wild-type cells. As expected, the HAT inhibitor A-485 inhibited the observed acetylation in both mutant cell lines (Fig. 8b).

We then forced CREBBP wild-type (293T, HN31, and Detroit 562) cells to express full-length or a representative truncated inhibitory-region mutant (Q1773X). In all three lines, expression 
a.

HR

UM-SCC-22a

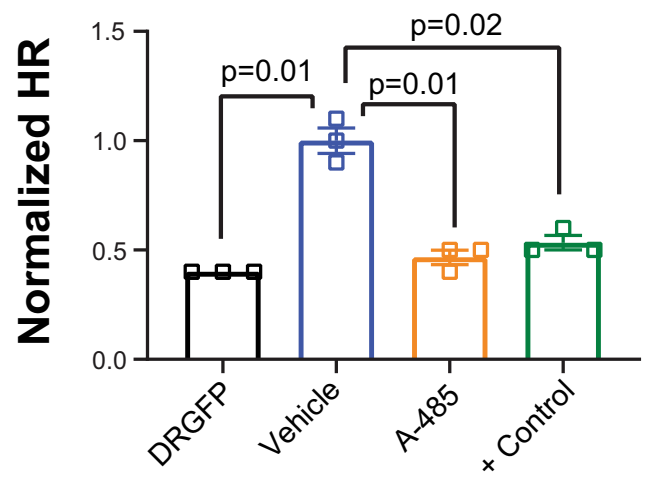

HN30

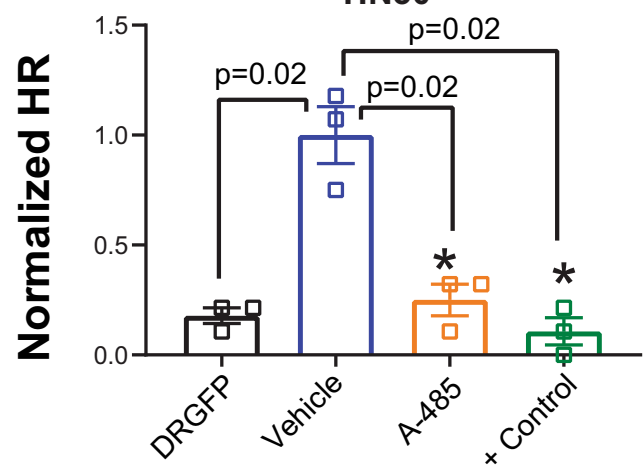

FaDu



HN31

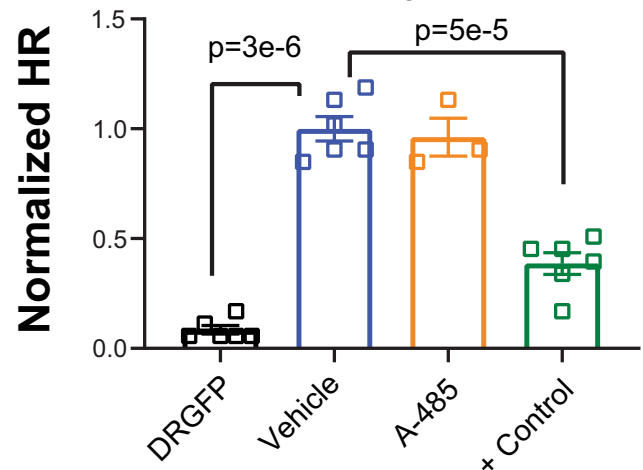

b.

NHEJ
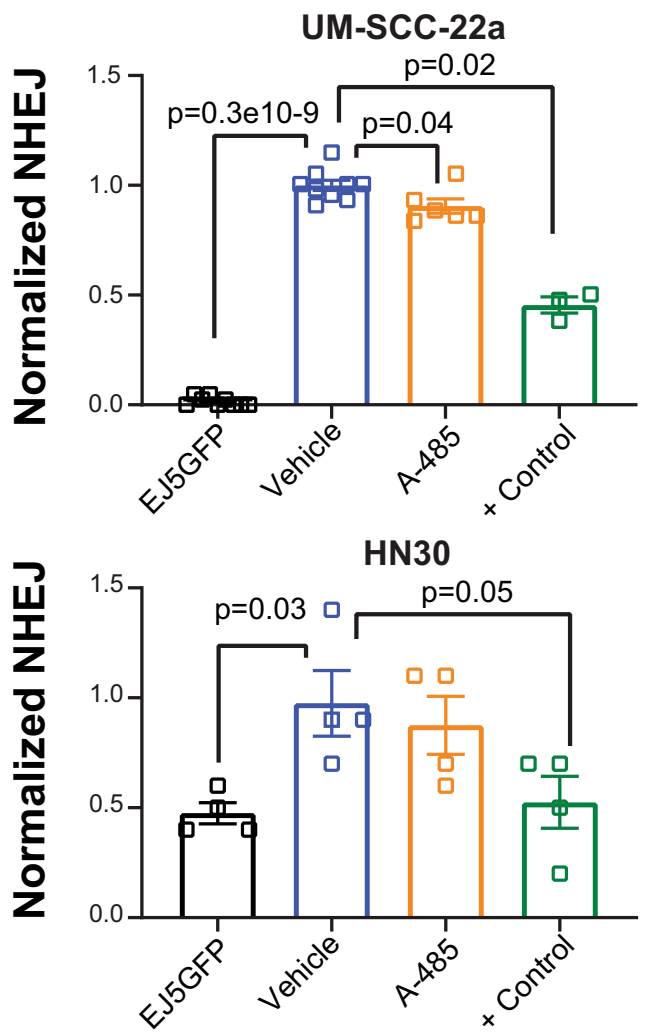

$\mathrm{FaDu}$
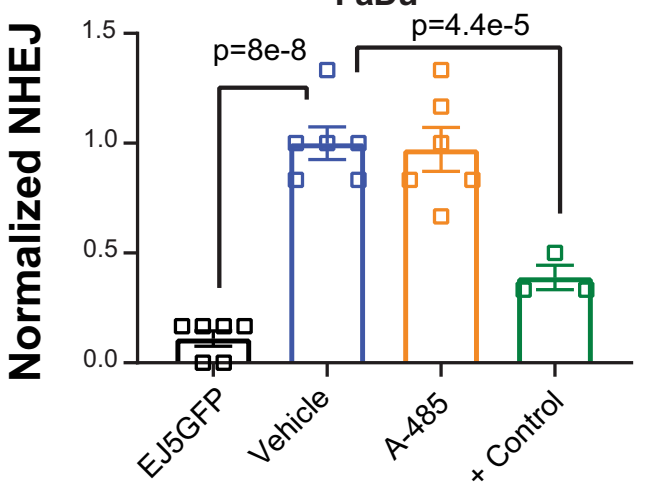

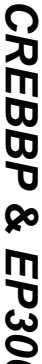

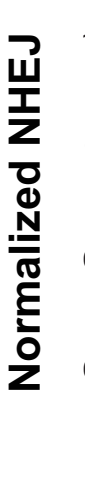



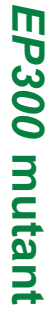

Fig. 6 HAT inhibition primarily reduces HR and not NHEJ in CREBBP/EP300 mutant cells. a, b I-Scel assay for HR (a) and NHEJ (b) as described in the methods following treatment with A-485, A-486, or + control (ATMi or ATRi). A minimum of three independent samples for each condition are shown and are presented as mean values +/-SEM. $p$-values for each indicated comparison are two-sided and derived from ANOVA with post hoc analysis adjusted for multiple comparisons. 
a.

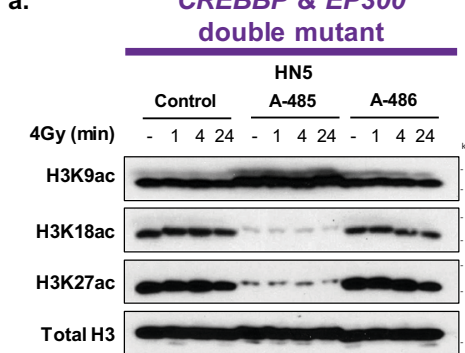

b.

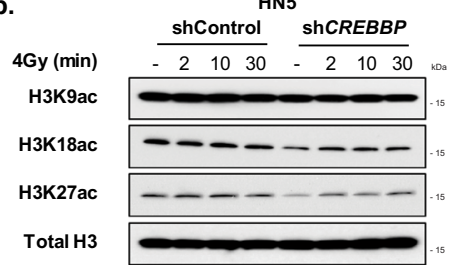

CREBBP mutant

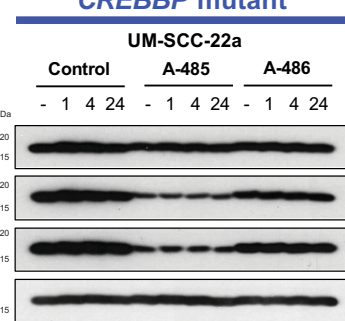

UM-SCC-22a

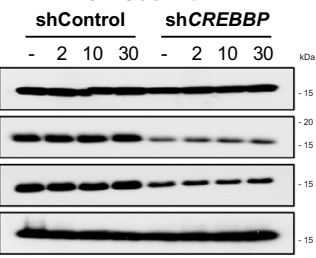

CREBBP \& EP300 wild type


c.

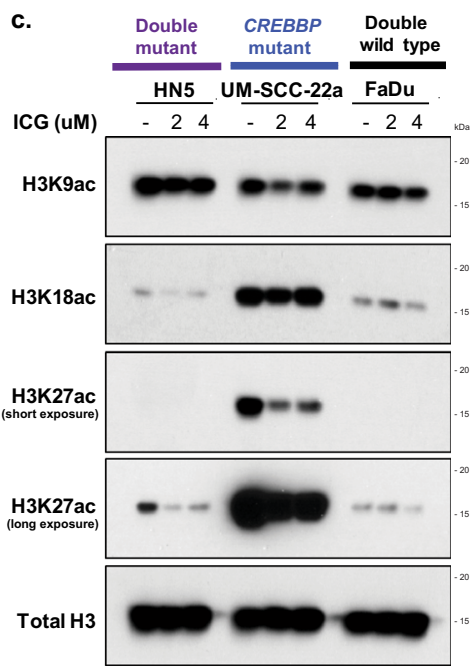

Fig. 7 Cells with mutations in CREBBP exhibit inhibition of histone acetylation following inhibition of CBP function. a-c Histone acid extraction and immunoblot following A-485 (a), shCREBBP (b), or ICG-001 (c) in HNSCC cell lines of varying CREBBP and EP300 status.

of the mutant led to a profound increase in $\mathrm{CBP}$ autoacetylation and global protein lysine acetylation that was not observed following forced expression of full-length CBP (Fig. 8c). Similarly, forced expression of mutant CBP led to increased acetylation at H3K18 and H3K27 histone marks (Fig. 8d). Additionally, we specifically examined the effects of mutant CBP on BRCA1 acetylation, which was similarly increased following forced expression of mutant CBP in HN31 and FaDu cell lines compared with wild type (Fig. 8e, f). Additionally, we examined the effects of forced expression of mutant CBP in both HN31 and FaDu cell lines on HR (Fig. 8g). In both cell lines, forced expression of mutant-but not wild-type-CBP led to significantly increased $\mathrm{HR}$, which was abrogated by A- 485 treatment.

Mutations in CREBBP/EP300 are associated with outcome following radiation in SCC. To examine the clinical association between CREBBP/EP300 mutation-and other mutations in HNSCC - with radioresponse, we identified a cohort of patients within the Head and Neck Cancer Genome Atlas (TCGA) that were denoted as having radiation as a component of their therapy (Supplementary Table 4). Genes significantly mutated at $\geq 10 \%$ frequency (high enough for potential clinical utility) were then queried to determine their relationship to overall survival (Fig. 9a). Only three genes (TP53 ( $p=0.015), C A S P 8(p=0.055)$, and $C R E B B P / E P 300(p=0.046))$ were associated with OS, as was the presence of HPV $(p=0.002)$ (47 patients, 17.4\%) (Fig. 9a). Tumor stage $(p=0.69)$, nodal stage $(p=0.91)$, and tumor site $(p=0.79)$ were not significantly associated with survival in this population, this is likely due to the similar clinical characteristics of the selected cohort, all of whom had advanced-stage disease treated with combined modality therapy. While TP53 was associated with OS in patients who did not receive radiation (HR 1.52, $p=0.051), C A S P 8(p=0.41)$ and $C R E B B P / E P 300(p=0.89)$ were not, indicating that in the latter genes, this phenomenon may be dependent upon radiation response.

Because the TCGA includes a cohort of patients with heterogeneous treatments and without details of patterns of failure (particularly the difference between LRR and DM), we examined patient outcomes in a more uniformly treated cohort of patients within this group, in which treatment and patterns of failure details were enumerated (Fig. 9b, c). This subset included 94 patients with HPV-negative HNSCC treated uniformly with surgery and postoperative radiation (clinical characteristics in
Supplementary Table 5). In this analysis, TP53 (as a binary variable), was not associated with OS or LRR (Fig. 9b, c). Mutations in both CASP8 and CREBBP/EP300 were associated with significantly reduced overall survival and higher rates of LRR in this patient population (Fig. 9b-e).

Since CREBBP/EP300 are commonly mutated in squamous tumors ${ }^{27}$, we expanded our analysis to other SCCs to determine whether their mutation might also be associated with poor outcome in other SCC tumor types treated with radiation. Among the TCGA squamous tumors, both lung and cervix had sufficient numbers and treatment annotations for analysis (clinical characteristics in Supplementary Table 6). We found that CREBBP/EP300 mutations were associated with poorer overall survival in radiation-treated patients with lung and cervix squamous tumors (Fig. 9f).

\section{Discussion}

There are no biologically driven precision-medicine approaches to radiation therapy, with treatment largely guided by clinical stage and intensified via the addition of cytotoxic chemotherapy. This leads to high degrees of toxicity as well as both over- and undertreatment, depending upon the patient and tumor. HNSCC is no exception to this phenomenon, with a highly toxic standard treatment of concurrent chemoradiation that has largely remained unchanged for decades. To improve this paradigm, we performed in vivo screening of HNSCC models and identified both general radiosensitizing targets and a genomically associated sensitization. This latter effect is potentially related to a gain of function in mutant CBP and p300, leading to increased basal acetylation and BRCA1 function, rendering these tumors highly sensitive to the combination of HAT inhibition and radiation (potential mechanism in Fig. 10).

In this work, we performed an in vivo shRNA screen for targets associated with radiosensitization. We chose HNSCC as our initial model, both due to the primacy of radiation in curative therapy and the relative dearth of targetable genetic alterations in this malignancy. Importantly, the use of in vivo screening takes into account tumor bulk, metabolism, angiogenesis, and stromal interactions, which are not identified in in vitro screens. The identification of several genes identified as targets for clinical radiosensitization in HNSCC by our own group and othersnotably CHEK $1^{28,29}$, PIK3CA ${ }^{15,30}$, PTK2 $2^{16}$, and XIAP ${ }^{14}$-argues for the utility of this technique in identifying more relevant 
a.
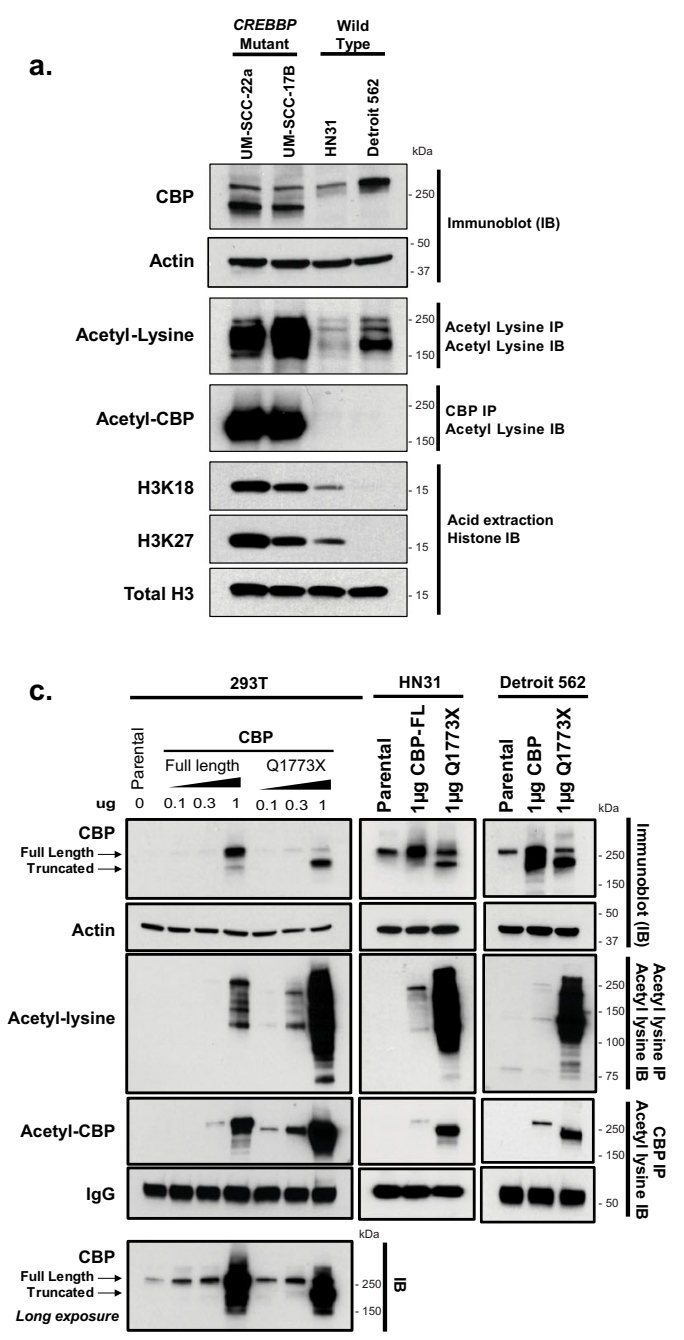

b.

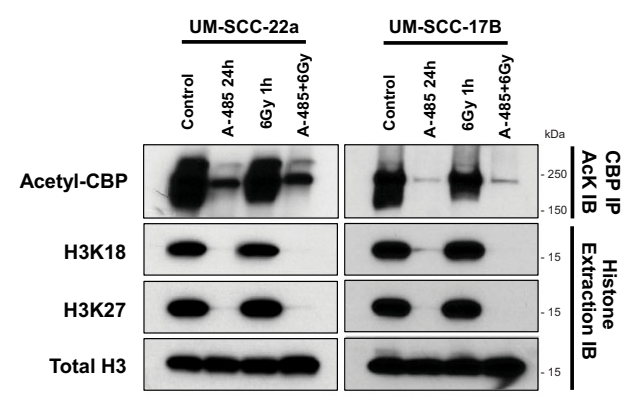

d.

d.

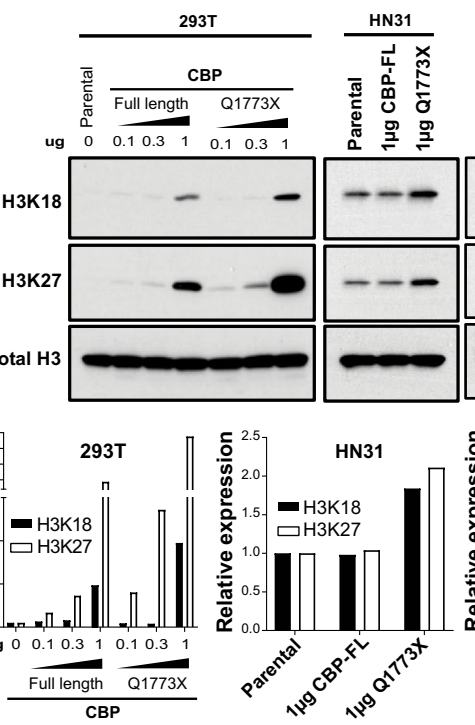

Detroit 562 e.

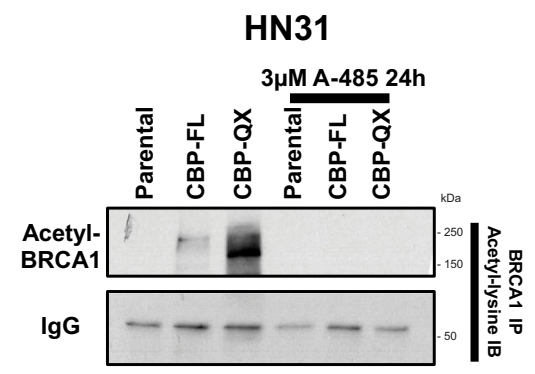

f.

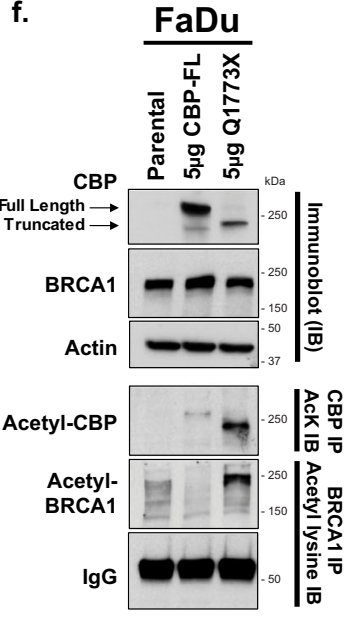

g.

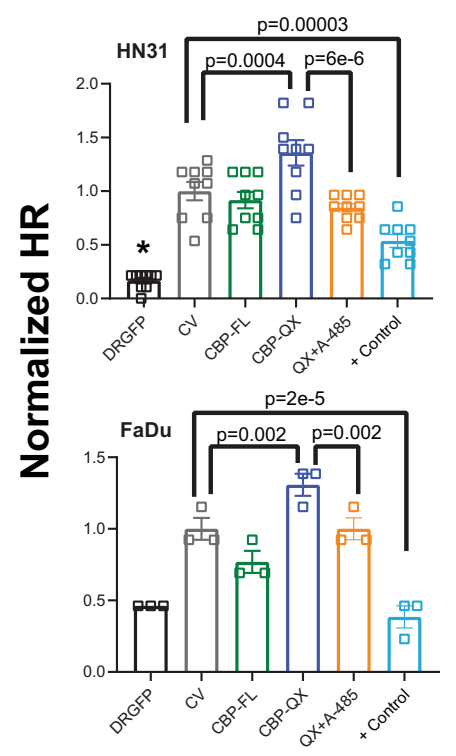

targets for clinical trial development. Validation of additional general targets for radiosensitization identified in a similar manner is ongoing.

However, despite the potential for these targets to improve response, toxicity remains a concern for agents, such as $\mathrm{PI} 3 \mathrm{~K}^{31,32}$ or $\mathrm{CHK} 1 / 2$ inhibitors ${ }^{33}$, which exert broad antitumor effects when they are combined with DNA-damage-based therapies in the clinic. One means of partially mitigating the overlapping toxicities of radiation and targeted therapies is tailoring of specific agents to genomic events that drive radioresistance. Evaluating 
Fig. 8 Gain-of-function mutation in CREBBP mutant cells. a-f Immunoblot (IB), histone acid extraction or immunoprecipitation (IP) for CBP, acetyl lysine, or BRCA1 was performed as indicated in the individual panels. a Global protein acetylation (IP acetyl lysine and IB for acetyl lysine), CBP autoacetylation (IP CBP and IB for acetyl lysine), and histone acetylation (acid extraction and IB) in HNSCC cell lines expressing wild-type CREBBP (HN31, Detroit 562) or CREBBP harboring a mutation in the TAZ2 domain (UM-SCC-22a, UM-SCC-17b). b CBP auto- and histone acetylation following treatment with A-485 in CREBBP mutant lines. c, d Global acetyl lysine and CBP autoacetylation (c), as well as histone acetylation (d) (densitometry below blot) in 293 T, HN31, and Detroit 562 cells forced to express either full-length CBP or a representative TAZ2 mutant CBP (Q1773X). e BRCA1 acetylation in HN31 cells forced to express full-length or TAZ2 mutant CBP and treated with A-485. f Total and acetyl CBP and BRCA1 in FaDu cells forced to express full-length or TAZ2 mutant CBP. $\mathbf{g}$ HR assay in either HN31 or FaDu cells forced to express full-length or TAZ2 mutant CBP. A minimum of three independent samples for each condition are shown and are presented as mean values $+/-$ SEM. Two-sided $p$-values for each indicated comparison are derived from ANOVA with post hoc analysis adjusted for multiple comparisons.

a.

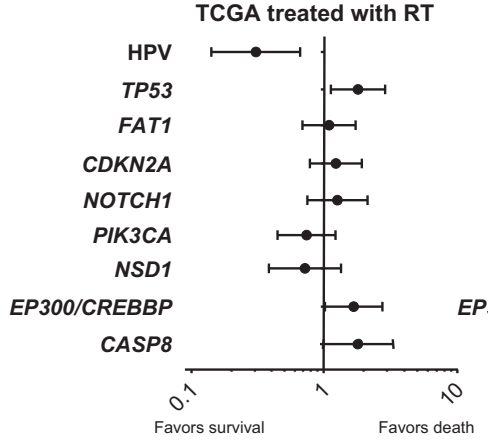

b.

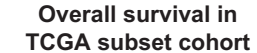

TP53

FAT1

CDKN2A

NOTCH1

PIK3CA

NSD1

EP300/CREBBP

CASP8

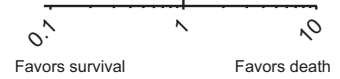

c.

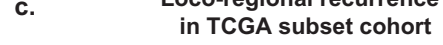

in TCGA subset cohort

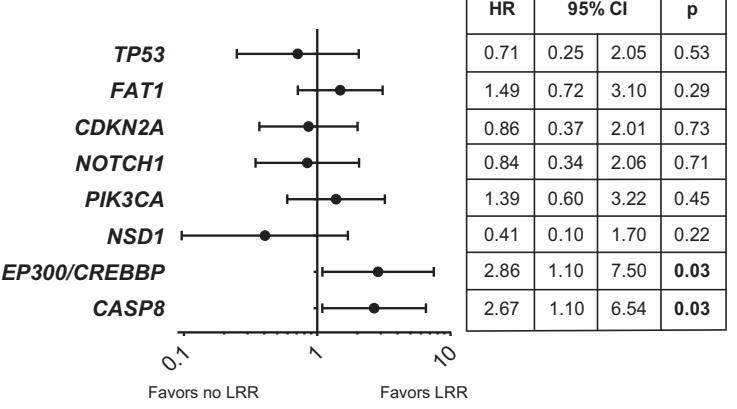

d.

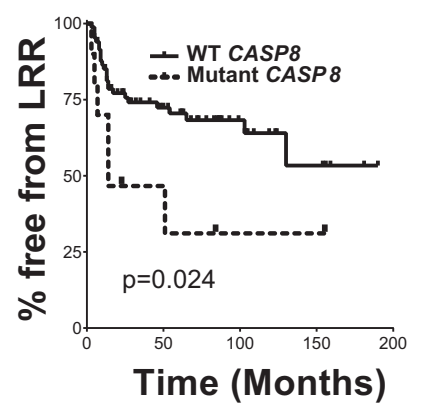

e.

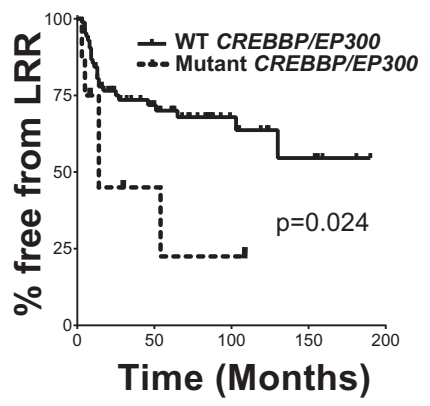

f.

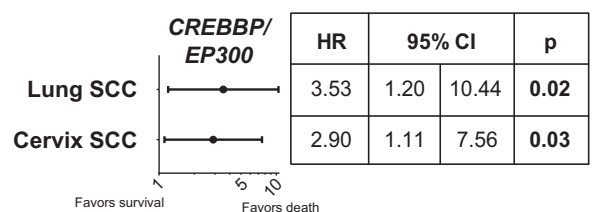

Lung SCC

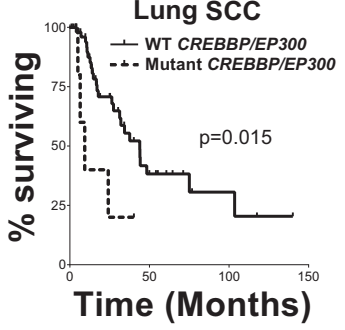

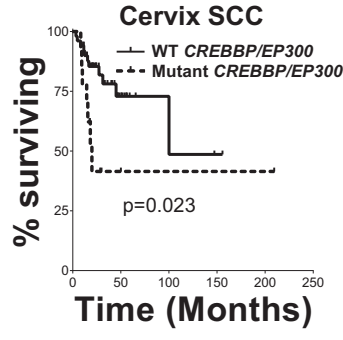

Fig. 9 Whole-exome sequencing identifies CREBBP/EP300 mutation significantly associated with outcome and treatment failure in several SCC cohorts. a Forrest plot of hazard ratios (HR) for overall survival (OS) for TCGA patients known to have received radiation therapy ( $n=276$ patients). b, c Forest plot of HR for OS (b) and locoregional recurrence (LRR) (c) in a subset of TCGA patients treated uniformly with surgery and radiation and known patterns of failure $(n=94$ patients). d, e Kaplan-Meier curves for LRR in patients with either CASP8 (d) or CREBBP/EP300 (e) mutations. f Forest plot and KM curves for OS by CREBBP/EP300 mutation in lung and cervix SCC cohorts.

our screening data in this manner, we found that inhibiting the protein-acetylation function of CBP and p300 led to a dramatic sensitization to radiation in CREBBP/EP300 mutant tumors and cell lines.

The concept of genomically driven radiation targeting is largely in its infancy. Despite ample evidence of genomic dependencies for targeted therapies - the classical example being PARP inhibition in $B R C A$-altered tumors ${ }^{12}$ - direct links between a particular somatic mutation or genetic alteration and sensitivity to the combination of radiation and a particular agent are limited. Indeed, most studies of radiosensitizers have focused broadly on agents that either affect DDR or inhibit kinases known to be important in tumorigenesis. This approach has the advantage of a potentially broad applicability but risks masking effects in specific groups of patients, ultimately leading to underperforming clinical trials and/or unacceptable toxicities. Conversely, identifying a specific effect in CREBBP/EP300 mutants, using a HAT inhibitor being developed for clinical use and radiation, can maximize response and tailor therapy to achieve a minimum of toxicity.

The identification of genomically associated radiosensitization in $C R E B B P / E P 300$ mutants is particularly of interest in HNSCC, and indeed in SCCs in general, as we have shown in the current study that mutations in these genes are associated with clinical radioresistance. This is the first large-scale examination of somatic mutations in this context and serves to link particularly treatment-resistant tumors with a genomically tailored therapy.

CREBBP and EP300 encode for homologous multifunctional bromodomain-containing acetyltransferases. Although these genes are mutated in HNSCC, they have not been extensively studied in this tumor type. Specifically, CREBBP and EP300 are collectively mutated in $13 \%$ of HNSCC $^{34}$, with similar frequencies in both HPV-positive and HPV-negative disease. Missense mutations are clustered in the acetyltransferase domain, and there is a reasonable frequency of truncating mutations ( 20\%). 


\section{Normal}



\section{Gain of function}

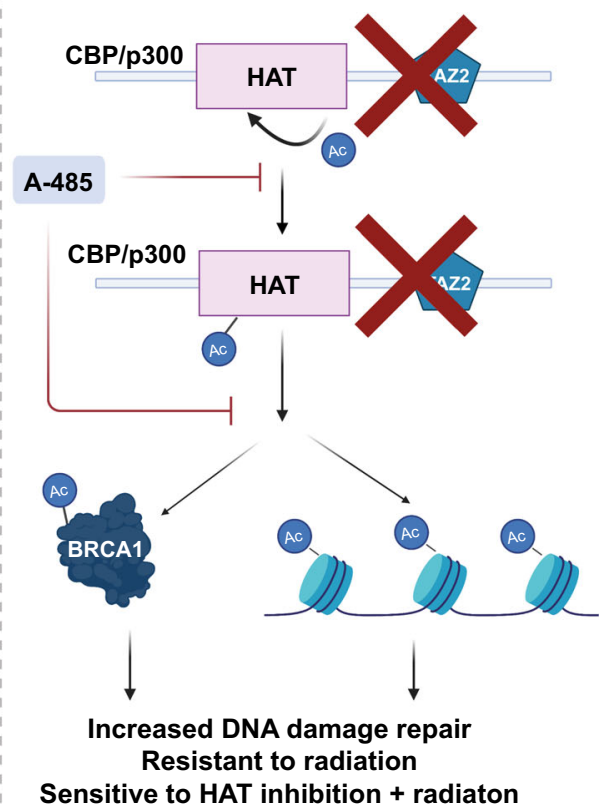

Fig. 10 Proposed mechanism of CREBBP/EP300 mutant-specific radiosensitization. Normal functioning of CBP and p300 shown on the left, with a potential gain of function (GOF) for both proteins shown on the right. The GOF mutation generally leads to increased protein acetylation, particularly BRCA1 acetylation, and increased DNA damage repair. Cells harboring these GOF mutations are sensitive to the combination of histone acetyltransferase (HAT) inhibition and radiation.

Additionally, many of these mutations are heterozygous, indicating possible haploinsufficiency, as has been seen for the chromatin-modifying genes in the BAF complex ${ }^{35}$. CREBBP and EP300 are also mutated in $14 \%$ of all squamous cancers ${ }^{27}$, and we found them associated with poor survival in lung and cervical tumors (Fig. 9f). Although much of our work has been done in HNSCC, it should be applicable to other tumor types, particularly squamous-cell carcinoma. Furthermore, it is possible that the mechanism can be expanded to include other acetylation or chromatin-modifying genes and shed light on the role of acetylation in DNA-damage response and repair.

Wild-type CBP and p300 are both known to localize to sites of DNA damage, but their roles and putative interaction partners at these sites remain undefined. Both proteins have been implicated in nonhomologous end joining and homologous recombination, although to what extent this is a reflection of their ability to bind and modify repair proteins as opposed to modifying surrounding chromatin is unclear. For example, both were found to bind to acetylated E2F1 and this binding was required for recruitment of CBP and p300 to the sites of DNA damage ${ }^{36}$, but the authors attributed this interaction to $\mathrm{CBP} / \mathrm{p} 300$ role in chromatin remodeling. Similarly, $\mathrm{CBP} / \mathrm{p} 300$ were found to acetylate $\mathrm{H} 3$ and $\mathrm{H} 4$ at sites of double-strand breaks, a chromatin modification that was required for the binding of KU70/80 to damaged $\mathrm{DNA}^{37}$. Counterintuitively, in HeLa and 293T cells, p300 was found to interact with chromodomain helicase DNA-binding protein 4 (CHD4) in order to promote homologous recombination but not nonhomologous end joining ${ }^{38}$, suggesting that the role of CBP and p300 in specific DNA double-strand break repair pathways may be context dependent.

Lending further support to the idea that $\mathrm{CBP} / \mathrm{p} 300$ play a role in homologous recombination, a recent report found that wildtype CBP/p300 promote transcription of BRCA1 and RAD51 ${ }^{39}$. Using HeLa cells and two lung-cancer cell lines, the authors showed that CBP and p300 physically bind to the promoter regions of both these genes, but also affect acetylation of histones $\mathrm{H} 3$ and H4, which led to insufficient binding of transcription factors. From this work, it was unclear to what extent CBP and p300 actively participate in transcriptional control or if their involvement was due to regional histone modifications that enable binding of transcriptional factors. The same study also identified CBP and p300 as the critical factors promoting RPA loading to DNA ends following BRCA1 resection, further lending support to the likelihood that both proteins extend their participation in homologous recombination via modifications to the nearby chromatin.

Aside from chromatin modifications, CBP/p300 were also found to acetylate RAD52, leading to RAD52 localization to double-strand breaks and subsequent RAD51 accumulation and repair via homologous recombination ${ }^{40}$. This CBP/p300-mediated RAD52 modification was dependent on ATM, connecting the first DNA-damage response steps via ATM sensing to downstream repair-complex assembly via RAD52. This suggests that both CBP and p300 can directly modify DNA-repair proteins potentiating their functions, implying that in certain situations, CBP and p300 may play a role in cancer resistance to DNAdamaging therapy. What remains unclear is if these activities are actively selected for during tumor progression, enabling tumors to indirectly hyperactivate DNA-repair pathways.

Previously, one study has identified an "addiction" to p300 in the context of CBP deletion, which was felt to replicate naturally occurring mutations in $C R E B B P^{41}$. However, in our study, we did not observe an effect with p300 inhibition in CREBBP mutant cell lines in our model, either at baseline or in combination with radiation. We also did not observe the converse in EP300 cell lines when CBP was inhibited. Thus, deletion of either protein may not recapitulate naturally occurring mutation.

Indeed, the data support a potentially more complex hypothesis of a gain of function for at least some mutations in $C R E B B P / E P 300$. Although we did not observe the effects on 
BRCA1 transcription in either wild type or mutant cells following modulation of CBP, we did identify high basal levels of both protein-including BRCA1-and histone acetylation in cell lines harboring mutations, which truncate the protein downstream of the HAT domain, with forced expression of similar mutations in wild-type cell lines recapitulating this effect. This acetylation is reversed following inhibition of CBP and p300 HAT activity.

A gain of function for CBP and p300 is not wholly unprecedented, as previous data from the Cancer Cell Line Encyclopedia suggested a gain of function for certain CREBBP mutants, specifically truncation mutations located in the TAZ domain, for acetylation at certain histone marks, although this was only identified in a few cell lines ${ }^{26}$. However, based on our data, this gain of function-albeit with varying degrees of basal activitymay be more prevalent than previously appreciated. Moreover, this gain of function appears to extend to both increased autoacetylation and acetylation of additional proteins, notably BRCA1. This would be in addition to a basal increase in acetylation of histone tails (H3K18, H3K27, and H4K5/8/12/16), which serves to allow DDR proteins to access damaged DNA and facilitate its repair more easily ${ }^{36,42}$. We believe that this state functions to generally promote a state primed to repair DNA via increased BRCA1 activity and HR, compared with cell lines lacking these mutations. This state, in turn, renders the combination of HAT inhibition and radiation highly lethal, via an inhibition of HR (see Fig. 10).

This study is limited in that the full spectrum of mutations in CREBBP and EP300 has not been studied. It is possible that the observed sensitization could be due to a combination of factors related to both a gain and loss of function for these proteins, particularly as the effect of missense mutations in the HAT domain, which are a significant proportion of all CREBBP/EP300 mutations, is unclear. Similarly, we are not certain about the most appropriate terminology to use for this phenomenon. Our screen was analyzed to identify genomically associated sensitization, with several targets identified. However, the relationship between $C R E B B P / E P 300$ mutations and genomically dependent targeting data is something akin to context-dependent oncogene addiction. However, many $C R E B B P / E P 300$ mutations have patterns consistent with loss of function, so more mechanistic studies are necessary to clarify the phenotype. Additionally, although we have identified BRCA1 and HR as likely mediators of this phenomenon, because of the relatively broad effects of the examined mutations on protein acetylation, additional studies are needed to determine if additional signaling pathways modulate this effect.

In conclusion, we have both identified prognostic markers of outcome following radiation in SCC and explored radiosensitization involving one of these biomarkers, mutated $C R E B B P / E P 300$. This genomically associated radiosensitization appears to specifically involve effects on DNA-damage repair, leading to a HR deficiency following DNA damage and leading to increased apoptosis. A gain-of-function effect in mutated cell lines may be driving this phenomenon, leading to a basal hyperacetylated state affecting BRCA1 function, which is abrogated using a HAT inhibitor. This agent is currently being explored for clinical trial use, and thus could be translated clinically to improve outcomes in SCC.

\section{Methods}

Cell lines and chemicals. HNSCC cell lines (UM-SCC-47, UM-SCC-22a, UMSCC-25, UM-SCC-1, HN31, HN30, UM-SCC-17B, UPCI:SCC-152, UD-SCC-2, Cal-27, and HN5) used in this study were generously supplied by Dr. Jeffrey Myers via The University of Texas MD Anderson Cancer Center Head and Neck cell line repository. HEK-293T, NCI-H520, NCI-H2228, NCI-H358, A549, Calu-6, FaDu, and Detroit 562 were purchased from American Type Culture Collection (Manassas, VA). Cell lines were tested for mycoplasma and genotyped before experiments.
UM-SCC-47, Cal 27, and UM-SCC-25 were maintained in Dulbecco modified Eagle medium (Gibco, USA), supplemented with $10 \%$ fetal bovine serum, $1 \%$ penicillin/streptomycin, $1 \%$ sodium pyruvate, $1 \%$ nonessential amino acids, and $2 \%$ vitamins. HEK-293T, HN5, HN30, HN31, UM-SCC-1, UM-SCC-17B, and UM-SCC-22a were maintained in DMEM/F-12 50/50 medium supplemented with $10 \%$ fetal bovine serum and $1 \%$ penicillin/streptomycin. UPCI:SCC-152, Detroit 562, Calu-6, and $\mathrm{FaDu}$ were maintained in MEM medium supplemented with $10 \%$ fetal bovine serum, $1 \%$ nonessential amino acids, and $1 \%$ penicillin/streptomycin. UD-SCC-2, NCI-H520, and NCI-H2228 were maintained in RPMI 1640 medium with $10 \%$ fetal bovine serum and $1 \%$ penicillin/streptomycin. All cell lines were incubated at $37^{\circ} \mathrm{C}$ and $5 \% \mathrm{CO} 2$ atmosphere.

ICG-001 was purchased from Selleck Chemicals (Houston, TX). A-485 and A-486 were manufactured by the MD Anderson Institute for Applied Cancer Science based on published structure ${ }^{23}$

Clonogenic survival assay. Single cells were plated in 6-well plates overnight. The next day, cells were incubated with specified drugs before irradiating at the indicated doses. The cells formed colonies over a 10- to 14-day incubation period and colonies were fixed in a $0.25 \%$ crystal violet/methanol solution. Colonies containing more than 50 cells each were counted. Survival curves were generated using GraphPad Prism (v8.0).

Immunoblot analysis. Cells were washed in PBS and scraped and collected in sufficient amount of whole-cell lysis buffer $(20 \mathrm{mM}$ HEPES, pH 7.9, $0.4 \mathrm{M} \mathrm{NaCl}$, $0.1 \mathrm{mM}$ EDTA, pH 8, $0.1 \mathrm{mM}$ EGTA, pH 7, 1\% Igepal, and 1X Halt proteaseinhibitor cocktail and 1X Halt phosphatase-inhibitor cocktail) (Thermo Sci). The lysate was mixed by vortexing and sonicated for $2 \mathrm{~min}$ at 100 amplitude with a QSonica Q700 sonicator (Newton, CT). Lysates were centrifuged at $20817 \times g$ for 15 min at $4{ }^{\circ} \mathrm{C}$. The supernatant was transferred to a fresh vial and total protein contents were estimated by DC Protein Assay kit (BioRad) and equal amounts of proteins were resolved on 4-15\% gradient (SDS)-polyacrylamide gel (Bio-Rad). The proteins were electrotransferred for $10 \mathrm{~min}$ onto polyvinylidene-difluoride (PVDF) membrane using Transblot Turbo device (Bio-Rad). After blocking with $5 \%$ nonfat powdered milk in Tris-buffered saline (TBS, $0.1 \mathrm{M}, \mathrm{pH}=7.4$ ), blots were incubated with primary antibody at $4{ }^{\circ} \mathrm{C}$ overnight. The following primary antibodies were used: p300 (NM11)(1:1000, sc-32244) from Santa Cruz Biotechnology (Santa Cruz, CA); Acetyl-Lysine (RM101) from Abcam (Cambridge, United Kingdom) (1:500, ab190479); $\beta$-actin (C4) from MilliporeSigma (Burlington, MA) (1:10000, \#MAB1501); and CBP (D6C5, 1:2000, \#7389), H3K9Ac (C5B11, 1:5000, \#9649), H3K18Ac (D8Z5H, 1:5000, \#13998), H3K27Ac (D5E4, 1:5000, \#8173), total Histone 3 (D1H2, 1:5000, \#4499), and Cleaved Caspase-3 (5A1E, 1:500, \#9664) from Cell Signaling Technology (Danvers, MA). After each step, blots were washed three times with Tween $(0.1 \%)$-Tris-buffer saline (TBS-T) Goat anti-mouse (1:2000, \#NA931V) and anti-rabbit (1:2000, \#NA934V) secondary antibodies conjugated to horseradish peroxidase (GE Healthcare, Chicago, Illinois) were used, and the signal was generated with the ECL2 western blotting substrate (Pierce Biotechnology, Rockford, IL) on HyBlot CL autoradiographic film (Thomas Scientific, Swedesboro, NJ). Protein abundance of $\beta$-actin served as a control for protein loading in each lane.

Immunoprecipitation. Cells were grown in 15-cm dishes until $75 \%$ confluency and lysed using $1 \mathrm{ml}$ of Pierce IP lysis buffer (Thermo) containing protease and phosphatase inhibitors. Lysates were sonicated for $2 \mathrm{~min}$ at $100 \%$ amplitude and cell debris was removed by centrifugation at $20817 \times \mathrm{g}$ for $15 \mathrm{~min}$. Protein quantification was estimated using DC protein assay kit. About $500 \mu \mathrm{g}$ of each sample were immunoprecipitated with $5 \mu \mathrm{l} \mathrm{CBP}$ antibody CBP (D6C5, 1:2000, \#7389) (Cell Signaling Tech, Danvers, MA), $25 \mu \mathrm{l}$ of BRCA1 antibody (D-9, 1:200, sc-9654) (Santa Cruz, CA), or anti-acetyl lysine affinity beads (AAC04-beads, Cytoskeleton Inc.), and incubated overnight rotating at $4{ }^{\circ} \mathrm{C}$. For CBP and BRCA1 IP, $50 \mu \mathrm{l}$ of $100 \mathrm{mg} / \mathrm{ml}$ Protein A Sepharose beads (17-0780-01, GE Healthcare) were added and rotated at $4{ }^{\circ} \mathrm{C}$ for $2 \mathrm{~h}$ the following day. Three $1-\mathrm{ml}$ washes with IP lysis buffer were used to isolate the precipitate and samples were boiled in $25 \mu \mathrm{l}$ of $2 \mathrm{X}$ SDSloading buffer for $7 \mathrm{~min}$ and loaded into $4-15 \%$ polyacrylamide gels (BioRad).

In vivo shRNA screen. Each library was cloned into the pRSI17 vector (Cellecta) and packed into lentivirus particles. HNSCC cell lines were infected in vitro through spinfection with virus containing the library at a low MOI ( $20 \%$ infected cells as measured by flow cytometry) in order to minimize superinfection of cells. Cells were selected with puromycin for at least two days and grown in vitro for $<3$ population doublings prior to injection of 4 million cells subcutaneously in nude mouse flank. An additional 2 million cells from the day of injection were collected as a frozen reference-cell pellet. Pilot studies were performed to (i) examine the frequency of tumor initiating cells (TIC) and determine whether the cell line could maintain shRNA-library complexity in vivo and (ii) identify the dose of radiation needed to achieve $\sim 20 \%$ tumor reduction for each model by the conclusion of the experiment.

For the screening experiment itself (and all other in vivo experiments), nude mice were housed between 68 and $79^{\circ} \mathrm{F}$, with $30-70 \%$ relative humidity and a $12 \mathrm{~h}: 12 \mathrm{~h}$ light:dark cycle. Xenografts were treated with $2 \mathrm{~Gy} /$ day of radiation once 
the tumor had reached approximately $100 \mathrm{~mm}^{3}$ to a total dose of 6-10 Gy, depending upon the model. Following treatment, the tumors were allowed to grow for approximately two weeks (volume $\sim 500 \mathrm{~mm}^{3}$ ). DNA was isolated from tumor and reference cells, amplified, and sequenced on Illumina sequencers ${ }^{43}$.

Hairpin counts were normalized to counts per million (CPM) per sample to enable comparison across samples. For each sample, $(\log 2)$ fold-change of each hairpin in the tumor was calculated compared with the level in the reference pellet. A hairpin summary measure per cell line was derived from the median of quantiletransformed $\log 2$ FC across replicates. Next, a modified version of the redundant siRNA-activity (RSA) algorithm ${ }^{44}$ was used to derive a gene-level summary measure per cell line. RSA attempts to provide a gene level summary estimate of the impact of knockout of the gene by calculating a stepwise hypergeometric test for each hairpin in a gene. Similar to GSEA, it is based on evidence from multiple hairpins of a gene showing an impact of cellular fitness. Our modifications were to ensure both that at least two hairpins were used when calculating the minimum $p$ value (in RSA) and that hairpins ranking above luciferase controls were not used when determining the minimum $p$-value. Quantile rank of luciferase-control barcodes was determined through evaluation across all experiments; on an average luciferase barcodes ranked $>0.6$ on the quantile-transformed $\log 2 \mathrm{fc}$ scale, so hairpins with quantile-transformed $\log 2 \mathrm{fc}>0.6$ were not used for the gene-level RSA score. Data displayed graphically using JMP Pro (v14) and GraphPad Prism (v8.0).

TUNEL assay. Following experimental treatments, all cells were collected, including floating cells, and TUNEL staining was performed using the APODIRECT Kit (BD Pharmingen) according to the manufacturer's protocol. Briefly, 500,000 cells were fixed in $1 \%$ paraformaldehyde on ice for $30 \mathrm{~min}$. Cells were then washed in PBS and fixed in $70 \%$ ethanol overnight at $-20 \mathrm{C}$. Cells were washed twice with provided buffer and then stained with $50 \mu$ of DNA-labeling solution at $37^{\circ} \mathrm{C}$ for $45-60 \mathrm{~min}$. Cells were then rinsed twice with provided buffer and resuspended in 300ul of rinse buffer. Cells were then analyzed by flow cytometry using the BD Accuri C6 flow cytometer (BD Biosciences) with 488-nm laser, 533/ 30 filter, and FL1 detector. In total, 10,000 events were measured per sample. Standard SSC and FSC gating were used to exclude debris. From the gated dot-plot display, additional gating was applied at the edge of the unstained cell population $(\sim 4 \log )$ and any events to the right of this population were gated as positive apoptosis $(\sim 5 \log )$. About $2 \mu \mathrm{g} / \mathrm{ml}$ puromycin 24 and $48 \mathrm{~h}$, in addition to kit controls, was used as positive-control samples to assist in proper delineation (exemplar gating shown in Supplementary Fig. 8a).

Cell cycle. About $24 \mathrm{~h}$ after irradiation, all cells were collected including floating cells. Cells were then pelleted by centrifugation at $300 \times g$ for $5 \mathrm{~min}$ and washed with PBS twice, then fixed using $70 \%$ ethanol for at least $30 \mathrm{~min}$ at $4{ }^{\circ} \mathrm{C}$ or overnight at $-20^{\circ} \mathrm{C}$. After fixation, cells were pelleted and washed once with PBS, then resuspended in propidium iodide $50 \mathrm{ug} / \mathrm{ml}$ (Sigma Aldrich), $100 \mathrm{ug} / \mathrm{ml}$ Rnase A (Sigma Aldrich) in PBS, and incubated at room temperature for $30 \mathrm{~min}$. Samples were then analyzed by flow cytometry using the Accuri C6 flow cytometer (BD Biosciences). Standard SSC and FSC gating were used to exclude debris. Standard gate was further gated by FL2-H and FL2-A, and additionally a third gating was applied, FL2-H by width, to remove doublets. A histogram was generated from these events and cell cycle distribution was quantified using FCS Express v7 using one-cycle DNA-fit analysis. Exemplar gating shown in Supplementary Fig. 8b.

RT-PCR. Cells were collected using RNA-extraction buffer and passed through a QIAshredder (Qiagen). Followed manufacturer's guidelines for RNA spin-column purification (RNeasy kit, Qiagen). Treated for $15 \mathrm{~min}$ at room temperature using DNaseI kit (Qiagen). Eluted total RNA with RNase-free water and quantified with nanodrop. Reverse-transcribed $1 \mu \mathrm{g}$ of total RNA into cDNA using iScript RT supermix (BioRad). PCR priming for $5 \mathrm{~min} 25^{\circ} \mathrm{C}$, RT $40 \mathrm{~min} 42^{\circ} \mathrm{C}$, inactivation $5 \mathrm{~min} 85^{\circ} \mathrm{C}$, and cool $\infty 8^{\circ} \mathrm{C}$ using T100 Thermal Cycler (BioRad). About $50 \mathrm{ng}$ of CDNA in triplicate was mixed with primers for BRCA1, CREBBP, or GAPDH (BioRad, PrimePCR) and iTaq Universal SYBR green supermix (BioRad). Samples were amplified and quantified using CFX Connect Real-Time PCR (BioRad) and analyzed using BioRad CFX Manager. Primers listed in Supplementary Table 7

Histone extraction. Histone proteins were extracted from treated or untreated cells using a histone-extraction kit (Abcam) according to the manufacturer's protocol. Briefly, cells were harvested, and the pellet was obtained by centrifugation at $10621 \times g$ for $5 \mathrm{~min}$ at $4{ }^{\circ} \mathrm{C}$. The cells were resuspended in $1 \mathrm{X}$ prelysis buffer and incubated at $4^{\circ} \mathrm{C}$ for $10 \mathrm{~min}$ on a rotator and then centrifuged for one minute at $10621 \times g$ at $4{ }^{\circ} \mathrm{C}$. The cell pellet was resuspended in lysis buffer at a concentration of $200 \mu \mathrm{L} / 10^{7}$ cells and incubated on ice for $30 \mathrm{~min}$, then centrifuged at $15294 \times g$ for $5 \mathrm{~min}$ at $4{ }^{\circ} \mathrm{C}$. The supernatant was collected and $300 \mu \mathrm{L}$ of balance buffer-DTT was added per $1 \mathrm{~mL}$ supernatant. The quantity of protein extracted was measured with a DC protein assay kit (Bio-Rad, Hercules, CA, USA). About $2-4 \mu \mathrm{g}$ of protein per sample was separated by western blot analysis as described previously.

Immunofluorescence staining. Immunofluorescence was performed to measure quantitative differences in DNA-damage repair and response. Cells were cultivated on cover slips placed in 35-mm cell culture dishes. At specified time points after exposure to radiation ( $2 \mathrm{~Gy}$ ), cells were fixed in $4 \%$ paraformaldehyde for $10 \mathrm{~min}$ at room temperature on a shaker, briefly washed in phosphate-buffered saline or PBS (BioRad), and placed in $70 \%$ ethanol at $4{ }^{\circ} \mathrm{C}$ overnight. Fixed cells were washed with PBS twice to remove ethanol and permeabilized with 0.1\% IGEPAL (octylphenoxypolyethoxyethanol) for $20 \mathrm{~min}$ at room temperature on a shaker, followed by blocking in $2 \%$ bovine serum albumin (Sigma) for $60 \mathrm{~min}$, and then incubated with anti- $\gamma \mathrm{H} 2 \mathrm{AX}$ (s139,1:200, \#2577), and 53BP1 (1:200, \#4937), both from Cell Signaling Tech, Danvers, MA or anti-BRCA1 primary antibody (D-9, 1:200,\#2577, Santa Cruz Technology, Santa Cruz, CA) overnight at $4{ }^{\circ} \mathrm{C}$. The next day, fixed cells were washed three times with PBS and incubated for 45 minutes in the dark in secondary anti-mouse antibody conjugated to FITC (1:600, \#715-165-150, Jackson ImmunoResearch, West Grove, PA) to visualize $\gamma \mathrm{H} 2 \mathrm{AX}$ or BRCA1. Secondary anti-rabbit antibody conjugated to Cy3 (1:600, \#711-165-152, Jackson ImmunoResearch, West Grove, PA) was used to visualize 53BP1. DNA was stained with $4^{\prime}, 6$-diamidino-2-phenylindole (Sigma) at 1:1000 $(1 \mu \mathrm{g} / \mathrm{ml})$. Immunoreactions were visualized with an Olympus or Leica Microsystems microscope (Wetzlar, Germany), and foci were counted with Image J software (https://imagej.nih.gov/ij/).

Plasmids, shRNA, and siRNA transfection. For siRNA, 1 million cells were transfected in $100 \mu \mathrm{l}$ of reagent $\mathrm{T}$ (Kit T, Lonza) using Nucleofector $2 \mathrm{~b}$ electroporator (Amaxa) program T-020, with 200nmol CREBBP siRNA (ON-TARGETplus, Horizon Discovery Biosciences). Human CREBBP siRNA:

GCACAGCCGUUUACCAUGA. Mock transfection was electroporated in $100 \mu \mathrm{l}$ of reagent T only. Cells were collected for mRNA and western blot analysis $48-64 \mathrm{~h}$ after transfection.

For shRNA, packaging cell line HEK-293T was cotransfected with $3 \mu \mathrm{g}$ of MISSION shRNAs specific for the CREBBP, EP300 gene, or control (Millipore/ Sigma) and lentiviral vectors DR8.2 and VSVG (Addgene). Two and three days after transfection, virus-containing media was filtered through a $0.45-\mu \mathrm{m}$ PVDF syringe filter and polybrene was added $(5 \mu \mathrm{g} / \mathrm{ml}$, Sigma). Target cells were transduced with virus for $4-6 \mathrm{~h}$ and were subjected to puromycin antibiotic selection. Pooled knockdown cells and counterpart shControl cells were assessed for CBP protein expression by immunoblotting. shRNA sequences are given as follows:

shRNA CREBBP 2\# TRCN0000006486 GCTATCAGAATAGGTATCATT

3\# TRCN0000006487GCGTTTACATAAACAAGGCAT

shRNA EP300 \#2 TRCN0000039884 5'-CCAGCCTCAAACTACAATAAA-3'

\#4 TRCN0000039886 5'-CCCGGTGAACTCTCCTATAAT-3'

\#5 TRCN0000039887 5'-CGAGTCTTCTTTCTGACTCAA-3'

Site-directed mutagenesis. Site-directed mutagenesis was performed using $50 \mathrm{ng}$ of KAT3A/CBP (CREBBP) (NM_004380) Human Tagged ORF Clone (OriGene) as the dsDNA template. This was carried out using the QuickChange II XL SiteDirected Mutagenesis Kit (Agilent Technologies) according to the manufacturer's protocol with the following exception: One Shot ${ }^{\text {mi }}$ Stbl $3^{\text {mi }}$ Chemically Competent E. coli (Invitrogen) was used for transformation rather than the XL10-Gold Ultracompetent Cells supplied with the kit because of the dependency on chloramphenicol selection already found in the full-length $C R E B B P$ vector. Mutagenic oligonucleotide primers were designed using Agilent QuickChange Primer Design program and purchased from Sigma-Aldrich with PAGE purification with the following sequences:

$5^{\prime}$-tggatgcagcgctagatgctcagccgg- $3^{\prime}$

$5^{\prime}$-ccggctgagcatctagcgctgcatcca- $3^{\prime}$

Following transformation, single colonies were selected on LB plates containing $34 \mathrm{ug} / \mathrm{mL}$ chloramphenicol, expanded in LB broth containing $34 \mathrm{ug} / \mathrm{mL}$ chloramphenicol overnight, and extracted with a QIAfilter midi kit (Qiagen). Sanger sequencing was utilized to confirm the presence of the desired mutation. Mutant plasmids were directly transfected into cell lines using GeneJet transfection reagent (SignaGen Labs) or packaged in $293 \mathrm{~T}$ for lentiviral infection.

HR/NHEJ repair assays. For GeneJet transfection, FaDu and HEK293T cells were plated into $10-\mathrm{cm}$ dishes. At $70 \%$ confluency, cells were transfected (GeneJet, SignaGen) with $5 \mu \mathrm{g}$ of pDRGFP (Addgene, Plasmid \#26475) or pimEJ5GFP (Addgene, Plasmid 44026) and stably selected with $2 \mu \mathrm{g} / \mathrm{ml}$ puromycin for two weeks. Stably selected cells were plated at 600,000 in $60-\mathrm{mm}$ dishes and incubated overnight. The next day, cells were treated with $1.5 \mu \mathrm{M}$ A-485/A-486, $10 \mu \mathrm{M} \mathrm{ATMi}$ (KU-55933), or $100 \mathrm{nM}$ ATRi (BAY-1895344) for $24 \mathrm{~h}$. The following day, cells were transfected with $3 \mu \mathrm{g}$ of pCBASceI (Addgene, Plasmid 26477) and $0.6 \mu \mathrm{g}$ of mCherry (Addgene, Plasmid 41583) and incubated with drugs for a total of $72 \mathrm{~h}$ Flow cytometry was run using BD Accuri C6 Plus and standard SSC and FSC gating excluded debris. A dot-plot display of FL1 (gfp) by FL2 (rfp) was gated at the edge of negative-control groups DRGFP or EJ5GFP. Any events to the right and upward from this gate were considered positive for repair (exemplar gating shown in Supplementary Fig. 8c). Data were plotted using GraphPad Prism v8.

For electroporation, UMSCC22A, HN31, HN30, A549, H460, Calu6, H520, H2228 and H358 $5 \mu \mathrm{g}$ of pDRGFP (Addgene, Plasmid \#26475), or pimEJ5GFP (Addgene, Plasmid 44026) were electroporated using Nucleofector $2 \mathrm{~b}$ technology (Amaxa) (see Supplementary Table 8 for programs used for individual cell lines) 
and stably selected with $2 \mu \mathrm{g} / \mathrm{ml}$ puromycin for two weeks. Stably selected cells were electroporated with $6 \mu \mathrm{g}$ of pCBASceI (Addgene, Plasmid 26477) and $2 \mu \mathrm{g}$ of mCherry (Addgene, Plasmid 41583) and incubated in $1.5 \mu \mathrm{M} \mathrm{A}-485 / \mathrm{A}-486,10 \mu \mathrm{M}$ ATMi (KU-55933), or $100 \mathrm{nM}$ ATRi (BAY-1895344) for a total of $72 \mathrm{~h}$. Flow cytometry was run using BD Accuri C6 Plus and standard SSC and FSC gating excluded debris. A dot-plot display of FL1 (gfp) by FL2 (rfp) was gated at the edge of negative-control groups DRGFP or EJ5GFP. Any events to the right and upward from this gate were considered positive for repair. Data were plotted using GraphPad Prism v8.

Mouse xenograft model. In vivo studies were performed according to all relevant ethical regulations and following Institutional Care and Use Committee (IACUC) approval from both the University of Pittsburgh and The University of Texas MD Anderson Cancer Center depending on the individual experiment. Male athymic nude mice (6-8-week old, ENVIGO/HARLAN, USA) were randomly assigned to treatment groups for each cell line tested (UM-SCC-47, UM-SCC-22a, and Detroit $562)$. Tumor cells $\left(2 \times 10^{6}\right.$ in $0.1 \mathrm{~mL}$ of serum-free medium) were injected subcutaneously in the right dorsal flank of each mouse. After palpable tumors had developed, tumor diameters were measured with digital calipers, and tumor volume was calculated as $\mathrm{A} \times \mathrm{B} 2 \times 0.5$, where $\mathrm{A}$ represents the largest diameter and $\mathrm{B}$ the smallest diameter. When the tumor volumes reached $\sim 150 \mathrm{~mm}^{3}$, tumors were irradiated with $16 \mathrm{~Gy}$ ( 2 Gy/day x 8 days) and tracked for approximately four weeks for tumor-growth delay experiments ( $n=\sim 7-10 /$ group). At that time, the experiment was completed, and tumors harvested. For in vivo TUNEL assay, tumors were collected $8 \mathrm{~h}$ after the last radiation treatment ( $n=5 /$ group). Days to reach $500 \mathrm{~mm}^{3}$ (UM-SCC-47 and Detroit 562) or $350 \mathrm{~mm}^{3}$ (UM-SCC-22a) for each group were calculated for each tumor and averaged within each treatment group rounded to the nearest day of measurement. Tumor volume was assessed at collection and averaged between groups. Kaplan-Meier curves were generated to evaluate survival, and group comparisons were performed using log-rank statistics.

In vivo TUNEL assay. Paraffin-embedded sections $(4 \mu \mathrm{m})$ of UM-SCC- 47 tumor xenografts were mounted on coated slides and sent to HistoWiz Inc. (histowiz.com) for TUNEL staining and quantification. TUNEL staining was performed using a standard operating procedure and fully automated workflow with Deadend colorimetric TUNEL system from Promega. After staining, sections were dehydrated and film coverslipped using a TissueTek-Prisma and Coverslipper (Sakura). Whole-slide scanning (40x) was performed on an Aperio AT2 (Leica Biosystems). Images were analyzed using Halo (version 2.3.2089.34) image analysis software from Indica Labs (Albuquerque, NM). Regions of interest were selected. TUNEL staining was segmented using the CytoNuclear algorithm. Total cell counts were thresholded into low-, medium-, and high-intensity staining bins.

Clinical data. This study was approved via the appropriate Institutional Review Board where applicable, complied with all relevant regulations regarding the use of human study participants, and was conducted in accordance with the criteria set by the Declaration of Helsinki. Informed consent was obtained for all participants in TCGA. The initial patient cohort consisted of the Head and Neck TCGA group that satisfied the following criteria: (i) whole-exome sequencing data are available and (ii) were denoted in the TCGA records as having received radiation as part of their initial therapy (clinical characteristics in Supplementary Table 4). Of the 523 patients in the TCGA cohort, a total of 276 patients met these criteria. Wholeexome sequencing from these tumors was examined for genes with mutations in $\geq 10 \%$ of tumors and significance on MutSig with the following genes meeting these criteria: TP53, FAT1, CDKN2A, NOTCH1, NSD1, CREBBP, and EP300 (combined due to significant homology), and CASP $8^{34,45}$. A subset $(n=94)$ of patients from this cohort have known specific treatment characteristics and patterns of failure analysis (clinical characteristics in Supplementary Table 5). These patients were all treated with surgery and post-operative radiation with well-annotated outcomes, including loco-regional recurrence and distant metastasis. In addition to the above, all tumors in this subset cohort were HPV/p16-negative.

Two additional cohorts from TCGA, the lung SCC and cervical SCC cohorts, were also examined, with a total of 61 and 66 patients respectively annotated as having received or who did likely receive (based on clinical scenario) external beam radiation and examined for outcomes (clinical characteristics in Supplementary Table 6).

For all clinical data, overall survival was defined from time of diagnosis until death or the last follow-up. Time to locoregional recurrence (LRR) or distant metastasis (DM) was defined as time from diagnosis until either an event or the last follow-up. Univariate analysis was performed using Cox regression (SPSS v25). Kaplan-Meier curves were generated, and group comparisons were performed using log-rank statistics.

Statistics and reproducibility. Comparisons between groups were performed using ANOVA with post hoc analysis adjusted for multiple comparisons (Graphpad Prism v8). A minimum of three biologic replicates were used for all in vitro experiments and representative figures were repeated at least twice with similar results as shown. The UM-SCC-47 in vivo tumor-growth delay study was performed twice with similar results in both experiments. The remaining in vivo studies were performed once.

Reporting summary. Further information on research design is available in the Nature Research Reporting Summary linked to this article.

\section{Data availability}

The data that support this study are available from the corresponding author upon reasonable request. Clinical outcome, tumor mutation, and gene expression data are publicly available from the Cancer Genome Atlas (TCGA) (https://gdac.broadinstitute org/, https://www.cbioportal.org/study/summary?id=hnsc_tcga, and https://portal.gdc. cancer.gov/projects/TCGA-HNSC) (dbGaP Study Accession phs000178). All protocols used in this study are available in the Methods or Supplementary Methods sections. Source data for figures in the report are available as a separate excel file. Source data are provided with this paper.

Received: 10 November 2020; Accepted: 12 October 2021; Published online: 03 November 2021

\section{References}

1. Barton, M. B. et al. Estimating the demand for radiotherapy from the evidence: a review of changes from 2003 to 2012. Radiother. Oncol. 112, 140-144 (2014).

2. Gillison, M. L. et al. Radiotherapy plus cetuximab or cisplatin in human papillomavirus-positive oropharyngeal cancer (NRG Oncology RTOG 1016): a randomised, multicentre, non-inferiority trial. Lancet 393, 40-50 (2019).

3. Mehanna, H. et al. Radiotherapy plus cisplatin or cetuximab in low-risk human papillomavirus-positive oropharyngeal cancer (De-ESCALaTE HPV): an open-label randomised controlled phase 3 trial. Lancet 393, 51-60 (2019).

4. Ang, K. K. et al. Randomized phase III trial of concurrent accelerated radiation plus cisplatin with or without cetuximab for stage III to IV head and neck carcinoma: RTOG 0522. J. Clin. Oncol. 32, 2940-2950 (2014).

5. Cohen, E. E. et al. Primary results of the phase III JAVELIN head \& neck 100 trial: Avelumab plus chemoradiotherapy (CRT) followed by avelumab maintenance vs CRT in patients with locally advanced squamous cell carcinoma of the head and neck (LA SCCHN). ESMO Virtual Congress 2020 (2020).

6. Pignon, J.-P., Maître, A., le, Maillard, E. \& Bourhis, J. Meta-analysis of chemotherapy in head and neck cancer (MACH-NC): An update on 93 randomised trials and 17,346 patients. Radiother. Oncol. 92, 4-14 (2009).

7. Bray, F. et al. Global cancer statistics 2018: GLOBOCAN estimates of incidence and mortality worldwide for 36 cancers in 185 countries. CA Cancer J. Clin. 68, 394-424 (2018).

8. Gillet, J.-P., Varma, S. \& Gottesman, M. M. The clinical relevance of cancer cell lines. J. Natl Cancer Inst. 105, 452-458 (2013).

9. Katt, M. E., Placone, A. L., Wong, A. D., Xu, Z. S. \& Searson, P. C. In Vitro Tumor Models: Advantages, Disadvantages, Variables, and Selecting the Right Platform. Front. Bioeng. Biotechnol. 4, 12 (2016).

10. Huang, A., Garraway, L. A., Ashworth, A. \& Weber, B. Synthetic lethality as an engine for cancer drug target discovery. Nat. Rev. Drug Discov. 19, 23-38 (2020).

11. Fong, P. C. et al. Inhibition of poly(ADP-ribose) polymerase in tumors from BRCA mutation carriers. N. Engl. J. Med. 361, 123-134 (2009).

12. O’Neil, N. J., Bailey, M. L. \& Hieter, P. Synthetic lethality and cancer. Nat. Rev. Genet. 18, 613-623 (2017).

13. Göttgens, E.-L. et al. Inhibition of CDK4/CDK6 enhances radiosensitivity of HPV negative head and neck squamous cell carcinomas. Int. J. Radiat. Oncol. Biol. Phys. 105, 548-558 (2019).

14. Xiao, R. et al. Dual antagonist of cIAP/XIAP ASTX660 sensitizes HPV- and $\mathrm{HPV}+$ head and neck cancers to TNFa, TRAIL, and radiation therapy. Clin. Cancer Res. 25, 6463-6474 (2019).

15. Skinner, H. D. et al. Integrative analysis identifies a novel AXL-PI3 KinasePD-L1 signaling axis associated with radiation resistance in head and neck cancer. Clin. Cancer Res. 23, 2713-2722 (2017).

16. Skinner, H. D. et al. Proteomic profiling identifies PTK2/FAK as a driver of radioresistance in HPV negative head and neck cancer. Clin. Cancer Res. https://doi.org/10.1158/1078-0432.CCR-15-2785 (2016)

17. Leiker, A. J. et al. Radiation enhancement of head and neck squamous cell carcinoma by the dual PI3K/mTOR inhibitor PF-05212384. Clin. Cancer Res. 21, 2792-2801 (2015).

18. Xie, T. et al. Abstract IA12: An in vivo high throughput shRNA screening platform for identifying ways to target genomic alterations. Clin. Cancer Res. 23, IA12-IA12 (2017)

19. Emami, K. H. et al. A small molecule inhibitor of beta-catenin/CREB-binding protein transcription [corrected]. Proc. Natl Acad. Sci. USA 101, 12682-12687 (2004). 
20. Wiese, $\mathrm{M}$. et al. The $\beta$-catenin/CBP-antagonist ICG-001 inhibits pediatric glioma tumorigenicity in a Wnt-independent manner. Oncotarget 8, 27300-27313 (2017).

21. Arensman, M. D. et al. The CREB-binding protein inhibitor ICG-001 suppresses pancreatic cancer growth. Mol. Cancer Ther. 13, 2303-14 (2014).

22. Crawford, T. D. et al. Discovery of a potent and selective in vivo probe (GNE-272) for the bromodomains of CBP/EP300. J. Med. Chem. 59, 10549-10563 (2016).

23. Lasko, L. M. et al. Discovery of a selective catalytic p300/CBP inhibitor that targets lineage-specific tumours. Nature 550, 128-132 (2017).

24. Liu, X. et al. BAP1 is a novel target in HPV-negative head and neck cancer. Clin. Cancer Res. 24, 600-607 (2018).

25. Kalu, N. N. et al. Genomic characterization of human papillomavirus-positive and -negative human squamous cell cancer cell lines. Oncotarget 8 , 86369-86383 (2017).

26. Ghandi, M. et al. Next-generation characterization of the cancer cell line encyclopedia. Nature 569, 503-508 (2019).

27. Liu, J. et al. An integrated TCGA pan-cancer clinical data resource to drive high-quality survival outcome analytics. Cell 173, 400-416.e11 (2018).

28. Barker, H. E. et al. CHK1 inhibition radiosensitizes head and neck cancers to paclitaxel-based chemoradiotherapy. Mol. Cancer Ther. 15, 2042-2054 (2016).

29. Zeng, L., Beggs, R. R., Cooper, T. S., Weaver, A. N. \& Yang, E. S. Combining Chk1/2 inhibition with cetuximab and radiation enhances in vitro and in vivo cytotoxicity in head and neck squamous cell carcinoma. Mol. Cancer Ther. 16, 591-600 (2017).

30. Marquard, F. E. \& Jücker, M. PI3K/AKT/mTOR signaling as a molecular target in head and neck cancer. Biochem. Pharmacol. 172, 113729 (2020).

31. Buchanan, C. M., Lee, K. L. \& Shepherd, P. R. For better or worse: the potential for dose limiting the on-target toxicity of PI 3-kinase inhibitors. Biomolecules 9, 402 (2019)

32. Hanker, A. B., Kaklamani, V. \& Arteaga, C. L. Challenges for the clinical development of PI3K inhibitors: strategies to improve their impact in solid tumors. Cancer Discov. 9, 482-491 (2019).

33. Soria, J. C. et al. A phase I, pharmacokinetic and pharmacodynamic study of GSK2256098, a focal adhesion kinase inhibitor, in patients with advanced solid tumors. Ann. Oncol. 27, 2268-2274 (2016).

34. Cancer Genome Atlas Network. Comprehensive genomic characterization of head and neck squamous cell carcinomas. Nature 517, 576-582 (2015).

35. Kadoch, C. \& Crabtree, G. R. Mammalian SWI/SNF chromatin remodeling complexes and cancer: mechanistic insights gained from human genomics. Sci. Adv. 1, e1500447 (2015)

36. Manickavinayaham, S. et al. E2F1 acetylation directs p300/CBP-mediated histone acetylation at DNA double-strand breaks to facilitate repair. Nat. Commun. 10, 4951 (2019).

37. Ogiwara, H. et al. Histone acetylation by CBP and p300 at double-strand break sites facilitates SWI/SNF chromatin remodeling and the recruitment of non-homologous end joining factors. Oncogene 30, 2135-2146 (2011).

38. Qi, W. et al. Acetyltransferase p300 collaborates with chromodomain helicase DNA-binding protein 4 (CHD4) to facilitate DNA double-strand break repair. Mutagenesis 31, 193-203 (2016).

39. Ogiwara, H. \& Kohno, T. CBP and p300 histone acetyltransferases contribute to homologous recombination by transcriptionally activating the BRCA1 and RAD51 genes. PLoS ONE 7, e52810 (2012).

40. Yasuda, T. et al. Novel function of HATs and HDACs in homologous recombination through acetylation of human RAD52 at double-strand break sites. PLoS Genet. 14, e1007277 (2018).

41. Ogiwara, H. et al. Targeting p300 addiction in CBP-deficient cancers causes synthetic lethality by apoptotic cell death due to abrogation of MYC expression. Cancer Discov. 6, 430-445 (2016).

42. Ziv, Y. et al. Chromatin relaxation in response to DNA double-strand breaks is modulated by a novel ATM- and KAP-1 dependent pathway. Nat. Cell Biol. 8, 870-876 (2006).
43. Carugo, A. et al. In Vivo functional platform targeting patient-derived xenografts identifies WDR5-Myc association as a critical determinant of pancreatic cancer. Cell Rep. 16, 133-147 (2016).

44. Birmingham, A. et al. Statistical methods for analysis of high-throughput RNA interference screens. Nat. Methods 6, 569-575 (2009).

45. Campbell, J. D. et al. Genomic, pathway network, and immunologic features distinguishing squamous carcinomas. Cell Rep. 23, 194-212.e6 (2018).

\section{Acknowledgements}

This work was supported by: (i) the National Cancer Institute R01CA168485-08 (HS) and P50CA097190-15 (RF), (ii) the National Institute for Dental and Craniofacial Research R01 DE028105 (HS) and R01DE028061 (HS and CP), and (iii) The Cancer Prevention Institute of Texas RP150293 (HS and CP). We thank Dr. Alma Faust for her assistance in editing this paper. Potential mechanism figure created with Biorender.com.

\section{Author contributions}

H.S. and C.P. directed the experiments and analyzed the clinical data. M.F. assisted in the planning, direction, and analysis of the in vivo screening experiments. M.K., T.X., L.Y., T.H., and S.S. directly performed the in vivo screening and/or processed and analyzed the resultant samples. M.K., K.B., J.M., A.H., and D.M. generated the stable K.D. cell lines. D.M., J.M., K.B., M.G., A.S., A.D., R.B., A.H., and M.K. performed the in vitro experiments. F.J., J.W., and L.S. generated and/or analyzed the HNSCC cell line RNA Seq and mutation data. B.B., J.M., and RF reviewed and evaluated the clinical data analysis. M.K. initiated this study, which was then continued by D.M. and J.M. This determined co-first author order.

\section{Competing interests}

No potential competing interests related to the paper are reported by any of the coauthors.

\section{Additional information}

Supplementary information The online version contains supplementary material available at https://doi.org/10.1038/s41467-021-26570-8.

Correspondence and requests for materials should be addressed to Heath D. Skinner.

Peer review information Nature Communications thanks Junran Zhang, Terence Williams, and the other, anonymous, reviewer(s) for their contribution to the peer review of this work.

Reprints and permission information is available at http://www.nature.com/reprints

Publisher's note Springer Nature remains neutral with regard to jurisdictional claims in published maps and institutional affiliations.

\section{(C) (1)}

Open Access This article is licensed under a Creative Commons Attribution 4.0 International License, which permits use, sharing, adaptation, distribution and reproduction in any medium or format, as long as you give appropriate credit to the original author(s) and the source, provide a link to the Creative Commons license, and indicate if changes were made. The images or other third party material in this article are included in the article's Creative Commons license, unless indicated otherwise in a credit line to the material. If material is not included in the article's Creative Commons license and your intended use is not permitted by statutory regulation or exceeds the permitted use, you will need to obtain permission directly from the copyright holder. To view a copy of this license, visit http://creativecommons.org/ licenses/by/4.0/.

(C) The Author(s) 2021 